\title{
联苯二酚的手性拆分及相转移催化不对称烷基化研究
}

\author{
柯翠连 $^{a} \quad$ 徐伟平 ${ }^{a} \quad$ 刘 达 ${ }^{a} \quad$ 刘 艳 ${ }^{*, a} \quad K^{2}$ Keiji Maruoka ${ }^{*, a, b}$ \\ $\left({ }^{a}\right.$ 广东工业大学轻工化工学院 广州 510006) \\ $\left({ }^{b}\right.$ Graduate School of Pharmaceutical Sciences, Kyoto University, Sakyo, Kyoto, 606-8501, Japan)
}

\begin{abstract}
摘要 手性相转移催化剂在手性药物、天然产物核心结构、手性功能分子构建中起着极其重要的作用, 因此近年来备 受关注. 以廉价易得的 $(R)-\alpha$-甲氧基苯乙酸为拆分剂与联苯衍生物进行酯化反应，成功地对联苯衍生物骨架进行了手性 拆分, 并基于拆分的光学纯 $C_{2}$ 轴手性联苯刚性骨架, 构建了系列新型联苯类相转移催化剂. 对该系列相转移催化剂进 行不对称催化烷基化反应, 产率高达 97\%，对映体选择性高达 $96 \%$. 对催化剂进行构效关系研究发现，对 2 位和 2'位的 羟基进行甲基化有利于催化反应的选择性，3 位和 $3^{\prime}$ 位引入叔丁基不利于反应的选择性. 催化剂经过结构优化后，在 5 位和 5'位引入 3,4,5-三氟苯基和 3,5-双 (三氟甲基)苯基都显示出较好的反应活性和选择性.
\end{abstract}

关键词 相转移催化; 联苯; 手性拆分; 不对称烷基化

\section{Chiral Resolution of Biphenol and Asymmetric Alkylation under Phase Transfer Catalysis}

\author{
Ke, Cuilian ${ }^{a}$ \\ $\mathrm{Xu}$, Weiping ${ }^{a}$ \\ Liu, $\mathrm{Da}^{a}$ \\ Liu, $\operatorname{Yan}^{*, a}$ \\ Maruoka, Keiji ${ }^{*, a, b}$ \\ ( ${ }^{a}$ School of Chemical Engineering and Light Industry, Guangdong University of Technology, Guangzhou 510006, China) \\ ( ${ }^{b}$ Graduate School of Pharmaceutical Sciences, Kyoto University, Sakyo, Kyoto, 606-8501, Japan)
}

\begin{abstract}
Chiral phase transfer catalysts play extrememly important role in the construction of natural products, the core structures of chiral drugs, and functional chiral chemicals, therefore have attracted more and more attention in recent years. In this paper, the chiral resolution of biphenyl skeletons was achieved by utilizing the readily available $(R)-\alpha$-methoxy benzene acetic acid as resolution agent. A series of new biphenyl type of phase transfer catalysts were designed and synthesized based on the optically pure $C_{2}$-symmetric chiral biphenyl framework. These catalysts are readily applicable to asymmetric alkylation of $N$-(diphenylmethylene)glycine tert-butyl ester with excellent enantioselectivity (up to 96\%) and yield (up to $97 \%$ ). The structure-activity relationship study on these catalysts showed that the methylation of hydroxyl group at C2 and C2' position is favorable for the selectivity, introduction of tert-butyl group at $\mathrm{C} 3$ and $\mathrm{C} 3$ ' position is unfavorable to both selectivity and reactivity. The catalysts bearing 3,4,5-trifluorophenyl group or 3,5-bis(trifluoromethyl)phenyl group at C5 and C5' position showed good reactivity and selectivity.
\end{abstract}

Keywords phase-transfer catalysis; biphenyl derivatives; chiral resolution; asymmetric alkylation

相转移催化(phase-transfer catalysis, PTC)因反应条 件温和、操作简单、环境友好、可使用水溶液以及易于 大量生产等优势, 在学术和工业生产中得到了广泛的应 用 ${ }^{[1-16]}$. 不对称相转移催化为手性药物、天然产物手性 核心结构、手性精细化学品等手性功能分子的构建提供 了方便有效的方法, 因此, 近年来备受关注 ${ }^{[1-21]}$. 手性 相转移催化剂在实现不对称相转移催化中发挥着极其
关键的作用. 我们在前期研究中开发了手性季铵盐类相 转移催化剂, 该类催化剂被命名为 Maruoka 相转移催化 剂[22-30]，包括基于手性联芸酚骨架的螺环型 Maruoka 相 转移催化剂 ${ }^{[21]}$, 基于手性联荎酚骨架的简化的 Maruoka 相转移催化剂, 以及基于手性联苯骨架的改良的 Maruoka 相转移催化剂, 如 Scheme 1 所示.

该系列相转移催化剂已经成功应用于催化不对称

\footnotetext{
* Corresponding authors. E-mail: yanliu@gdut.edu.cn; maruoka@kuchem.kyoto-u.ac.jp Reveived March 11, 2020; revised April 28, 2020; published online May 11, 2020.

Project supported by the National Natural Science Foundation of China (Nos. 21977019, 21502023), the Guangdong Provincial Key Research and Development Program (No. 2019B020201005), the Open Project of Guangdong Provincial Key Laboratory of New Drug Screening (No. GDKLNDS-2018OF004). 国家自然科学基金(Nos. 21977019, 21502023)、广东省重点领域研发计划(No. 2019B020201005)、广东省新药篮选重点实验室开放课题(No. GDKLNDS-2018OF004).
} 


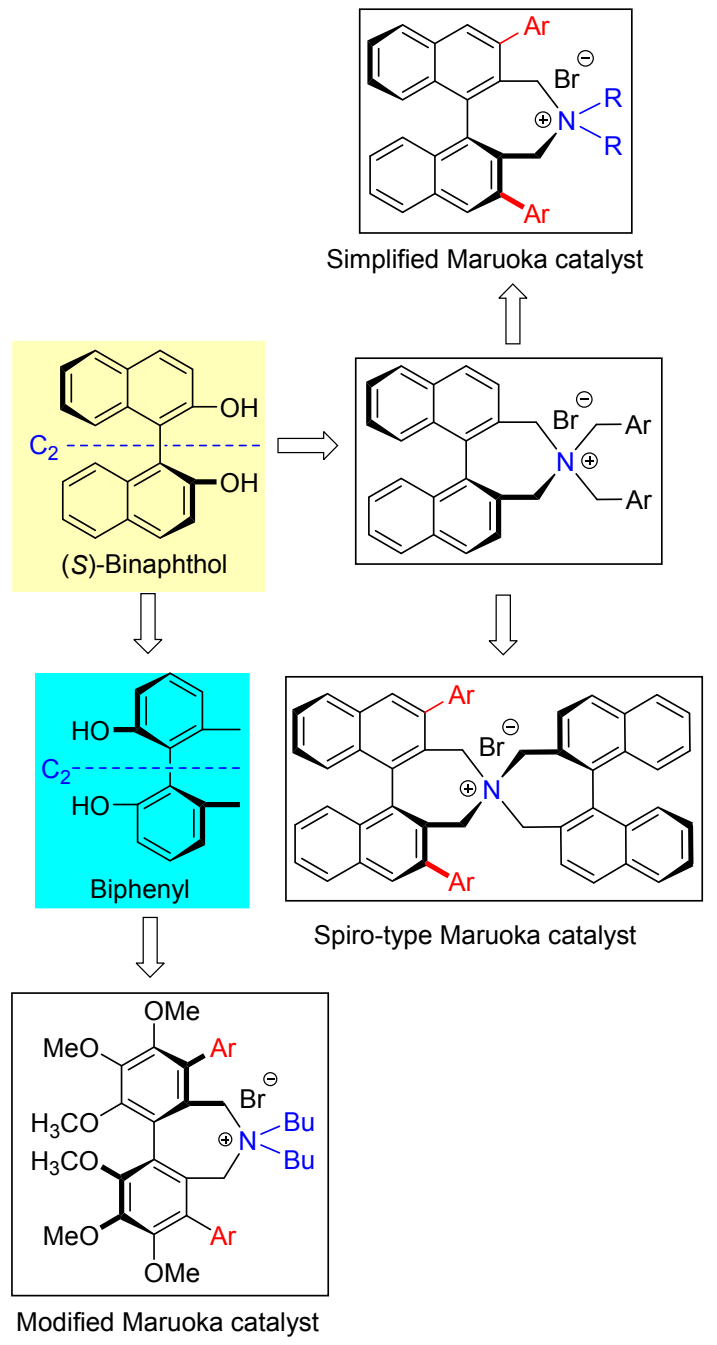

图式 1 Maruoka 相转移催化剂的结构

Scheme 1 Structure of Maruoka catalysts

烷基化反应 ${ }^{[21-23,25,29-36]}$ 、不对称偶联反应 ${ }^{[28,37-42]}$ 、不对称 曼尼希反应 ${ }^{[43-47]}$ 、不对称羟醛缩合反应 ${ }^{[48-50]}$ 、不对称环 化反应 ${ }^{[51-52]}$ 、不对称氰化反应 ${ }^{[53-54]}$ 等反应类型. 其中, 催化不对称烷基化反应已成功应用于开发天然氨基酸、

人造氨基酸和肽. 基于联苯骨架的改良 Maruoka 相转移 催化剂, 在不对称烷基化反应中表现出较高的催化活性 (0.01 1 mol\%的催化剂用量)和优异的立体选择性, 为 $\alpha$-烷基取代 $\alpha$-氨基酸的合成提供了实用的方法 ${ }^{[34-35,55-56]}$. 光学纯的联苯骨架对于手性联苯类相转移催化剂的开 发至关重要. 为了构建该类催化剂, 我们早期对联苯衍 生物 5,5'-二溴-3,3'-二叔丁基-6,6'-二甲基-2,2'-联苯酚进 行了手性拆分 ${ }^{[57]}$. 以 $(R, R)$-二苯基乙二胺为拆分剂, 通 过与轴手性的联苯酚消旋体进行成盐反应对其进行化 学拆分，拆分后的单一联苯衍生物的 ee 值 $>99 \%$ (Scheme 2a). 然而, $(R, R)$-二苯基乙二胺作为拆分剂价格 昂贵, 操作复杂. 因此, 为了获得光学纯的联苯类催化 剂骨架，急需开发更加实用的拆分方法. (a) Previous work

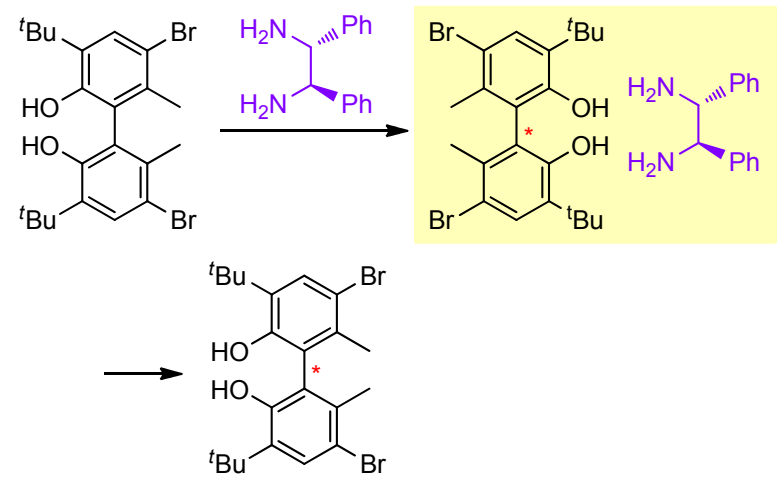

(b) This work

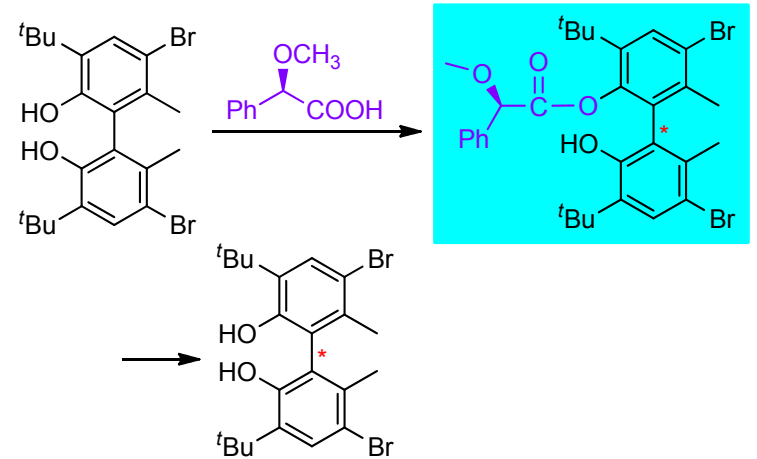

图式 2 轴手性联苯衍生物骨架的化学拆分方法

Scheme 2 Chiral resolution of the skeletons of axial chiral biphenyl derivatives

基于此，我们开发了基于廉价易得的 $(R)-\alpha$-甲氧基 苯乙酸为拆分剂的轴手性联苯骨架的化学拆分方法 (Scheme 2b).

另外，本工作基于拆分的光学纯的联苯衍生物骨 架，合成了系列新型联苯类相转移催化剂，将其应用于 催化不对称烷基化反应，并对催化剂的反应活性和选择 性进行了构效关系研究.

\section{1 结果与讨论}

\section{$1.12,2^{\prime}$-联苯衍生物的化学拆分}

轴手性联苯骨架是构建联苯类相转移催化剂的关 键结构单元. 联苯衍生物的化学拆分是获得轴手性骨架 的有效手段. 本工作以廉价易得的 2-叔丁基-5-甲基苯 酚为起始原料, 利用偶联反应获得联苯骨架，进而对其 手性拆分, 合成路线如 Scheme 2 所示. 以 2-叔丁基-5甲基苯酚 (1)为原料，在二氯甲烷和甲醇的混合溶剂中 采用四丁基三溴化铵为溴化剂，澳化得到 4-溴-2-叔丁 基-5-甲基苯酚(2), 收率为 99\%。在氯化亚铜催化下，对 化合物 2 进行氧化偶联反应得到消旋的联苯酚骨架 $\mathbf{3}$, 收率为 $59 \%$. 以廉价易得的 $(R)-\alpha$-甲氧基苯乙酸为化学 拆分剂，通过与消旋的联苯酚骨架 3 进行酯化反应，定 
量得到单酯化产物 $(R, S)$-4(收率为 $49 \%$ ) 和双酯化产物 $(R, R, R)-\mathbf{5}$ (收率为 $32 \%)$. 以乙酸乙酯/二氯甲烷/正己烷 (体积比 $1: 1: 20$ ) 为洗脱剂, 化合物 $(R, S)-\mathbf{4}$ 的 $R_{\mathrm{f}}$ 值为 0.5 , 化合物 $(R, R, R)-5$ 的 $R_{\mathrm{f}}$ 值为 0.3 . 化合物 $(R, S)-\mathbf{4}$ 和 $(R, R, R)-5$ 经 $\mathrm{LiAlH}_{4}$ 还原后得到光学纯的轴手性化合物 $(S)-3$ 和 $(R)-3$, 产率为 $96 \%, 0{ }^{\circ} \mathrm{C}$ 下在甲苯中通过三氟甲 磺酸对轴手性化合物(S)-3 和( $R$ )-3 进行脱除叔丁基反应, 得到光学纯的轴手性化合物 $(S)-6$ 和 $(R)-6$, 产率为 94\%.

\section{2 联苯类轴手性相转移催化剂制备}

在前期研究工作的基础上, 为了进一步研究联苯类 轴手性相转移催化剂催化不对称烷基化的反应活性和 选择性, 设计合成了 8 个催化剂以探讨催化剂催化不对 称烷基化反应的构效关系: (1) 2,2'位羟基对催化的影响, 设计合成了 2,2'位羟基和 $2,2^{\prime}$ 位甲氧基取代的催化剂; (2) $3,3^{\prime}$ 位取代基团的影响, 设计合成了 3,3'位叔丁基取代及 不含取代基的催化剂; (3) 5,5'位芳香基团的影响, 设计 合成了 5,5'位为 3,4,5-三氟苯基取代和 3,5-双 (三氟甲基) 苯基为取代基的催化剂. 以拆分得到的光学纯轴手性骨 架化合物 $(S)$-3 和(S)-6 为原料, 在乙二醇二甲醚(DME) 和水的混合体系中, $\mathrm{Pd}\left[\mathrm{P}(\mathrm{Ph})_{3}\right]_{4}$ 和 $\mathrm{Na}_{2} \mathrm{CO}_{3}$ 存在条件下, 与 3,5-双 (三氟甲基)苯嗍酸进行偶联反应, 得到催化剂 中间体 $(S)-7$ 和 $(S)-8$, 收率分别为 $97 \%$ 和 $94 \%$. 中间体
$(S)$-7 和 $(S)-8$ 在丙酮中以 $\mathrm{K}_{2} \mathrm{CO}_{3}$ 为缚酸剂, 与碘甲烷反 应, 将 2,2'位着基进行甲基化, 进一步得到催化剂中间 体 $(S)-9$ 和 $(S)-10$, 收率分别为 99\%和 99\%. 中间体 $(S)-9$ 和 $(S)-10$ 在苯中以偶氮二异丁腈(AIBN)为自由基引发 剂, $N$-溴代丁二酰亚胺(NBS)为溴化剂, 将 6,6'位甲基进 行溴化, 得到催化剂中间体 $(S)-11$ 和 $(S)-12$, 收率分别为 94\%和 96\%, 中间体 $(S)-11$ 和 $(S)-12$ 在乙腈中以 $\mathrm{K}_{2} \mathrm{CO}_{3}$ 为缚酸剂, 与二丁基胺反应得到催化剂(S)-13 和催化剂 $(S)-14$. 收率分别为 $66 \%$ 和 $82 \%$. 对催化剂 $(S)-13$ 和 (S)-14 的 2 位和 2'位去甲基化反应得到 2 位和 2'位羟基 的 $(S)-15$ 和 $(S)-16$ 催化剂, 收率分别为 $81 \%$ 和 $85 \%$. 该系 列催化剂在 5 位和 5 '位含有 3,5-双 (三氟甲基)苯基, 合成 路线如 Scheme 3 所示.

基于同样的合成方法, 以拆分得到的光学纯轴手性 骨架化合物 $(R)-3$ 和 $(R)-6$ 为原料, 在 5 位和 5 '位引入 3,4,5-三氟苯基取代基, 进一步合成了 3,4,5-三氟苯基取 代的相转移催化剂, 包括 2 位和 2 '位为甲氧基的催化剂 $(R)-23$ 和 $(R)-\mathbf{2 4}, 2$ 位和 $2^{\prime}$ 位为羟基的催化剂 $(R)-25$ 和 $(R)-26$, 合成路线如 Scheme 4 所示.

\section{3 催化不对称烷基化反应及催化剂的构效关系}

基于合成的 8 个联苯类相转移催化剂, 我们对催化 剂催化甘氨酸衍生物不对称烷基化反应的反应活性及
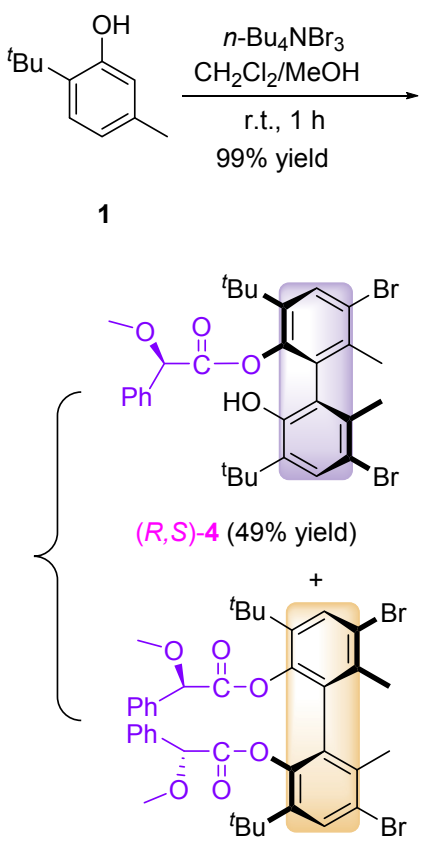

$(R, R, R)-5$ (32\% yield)
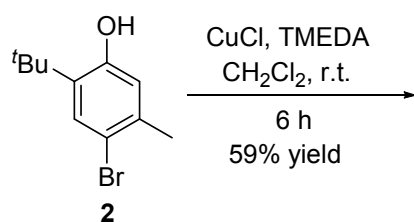

2

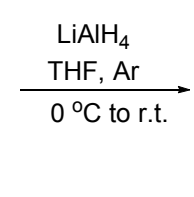

(S)-3 (96\% yield)
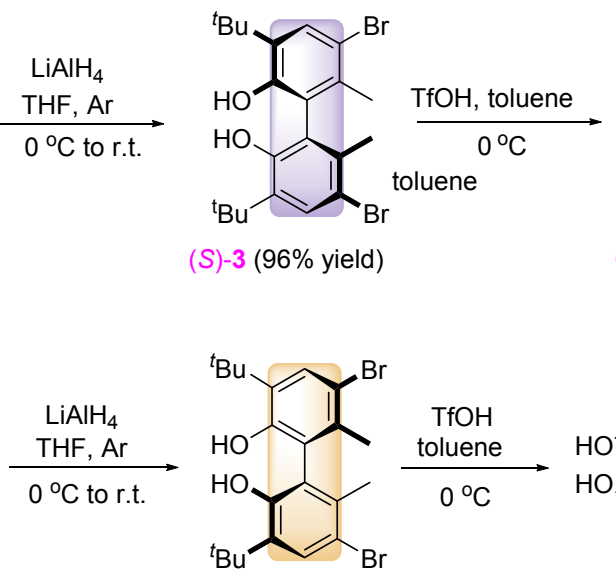

(R)-3 (96\% yield)

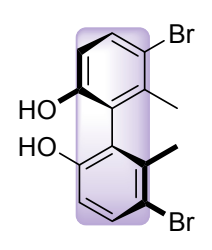

(S)-6 (94\% yield)

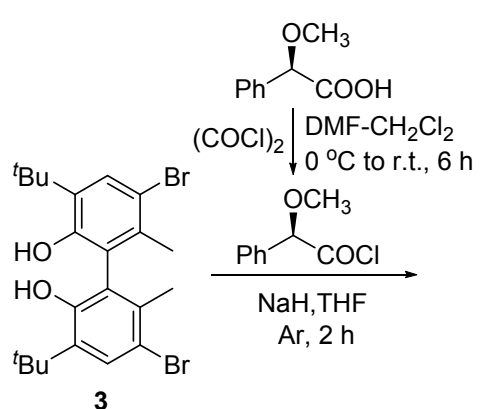

(S)
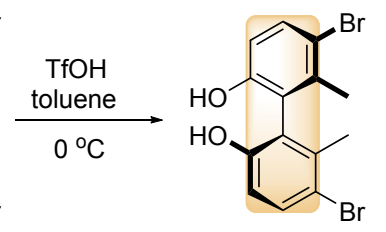

(R)-6 (94\% yield)

图式 2 2,2'-联苯酚衍生物的化学拆分

Scheme 2 Chiral resolution of 2,2'-diphenol derivatives 


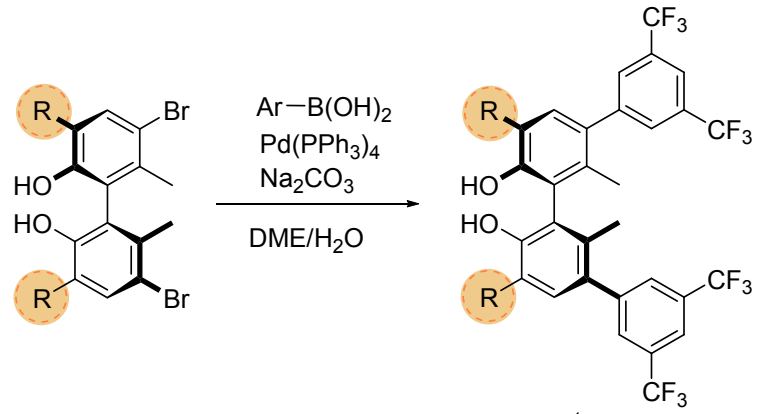

(S)-3: $R={ }^{t} \mathrm{Bu}$

$(S)-6: R=H$<smiles>[R]c1cc(-c2cc(C(F)(F)F)cc(C(F)(F)F)c2)c(CBr)c(-c2c(CBr)cc([2H])c(O)c2OC)c1-c1cc(C(F)(F)F)cc(C(F)(F)F)c1</smiles>

(S)-11: $R={ }^{t} \mathrm{Bu}, 94 \%$ yield (S)-12: $\mathrm{R}=\mathrm{H}, 96 \%$ yield
(S)-7: $\mathrm{R}={ }^{t} \mathrm{Bu}, 97 \%$ yield

(S)-8: $\mathrm{R}=\mathrm{H}, 94 \%$ yield

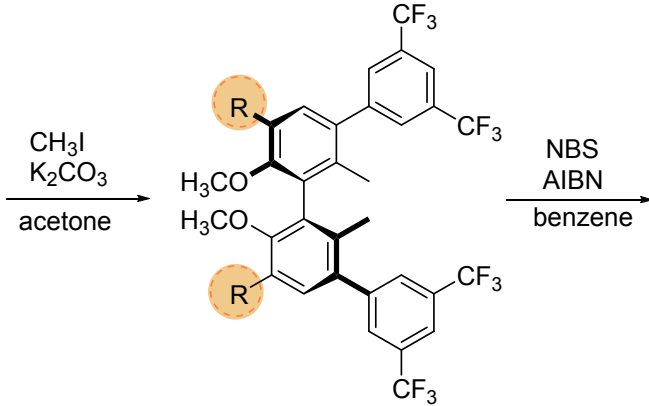

(S)-9: $\mathrm{R}={ }^{t} \mathrm{Bu}, 99 \%$ yield

(S)-10: $\mathrm{R}=\mathrm{H}, 99 \%$ yield
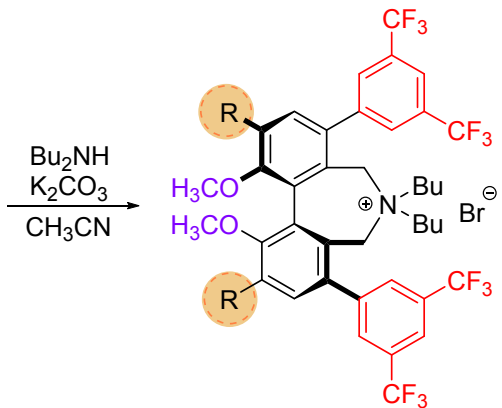

(S)-13: $\mathrm{R}={ }^{t} \mathrm{Bu}, 66 \%$ yield

(S)-14: $\mathrm{R}=\mathrm{H}, 82 \%$ yield

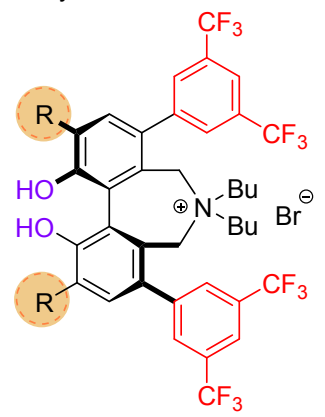

(S)-15: $\mathrm{R}={ }^{\mathrm{t}} \mathrm{Bu}, 81 \%$ yield

(S)-16: $\mathrm{R}=\mathrm{H}, 85 \%$ yield

图式 3 催化剂 $(S)-13,(S)-14,(S)-15$ 和 $(S)-16$ 的合成路线

Scheme 3 Synthetic route for catalysts $(S)-13,(S)-14,(S)-15$ and $(S)-16$

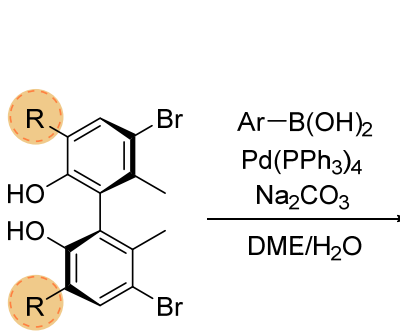

(R)-3: $\mathrm{R}={ }^{\mathrm{t}} \mathrm{Bu}$ $(R)-6: \mathrm{R}=\mathrm{H}$

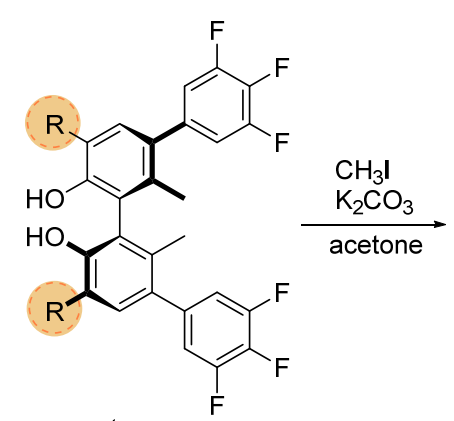

(R)-17: $\mathrm{R}={ }^{\mathrm{t}} \mathrm{Bu}, 93 \%$ yield (R)-18: $\mathrm{R}=\mathrm{H}, 95 \%$ yield<smiles>[R]c1cc(-c2cc(F)c(F)c(F)c2)c(C)c(-c2c(C)c([R])cc(-c3cc(F)c(F)c(F)c3)c2OC)c1C</smiles>

$(R)-19: \mathrm{R}={ }^{t} \mathrm{Bu}, 95 \%$ yield $(R)-20: \mathrm{R}=\mathrm{H}, 99 \%$ yield<smiles>[R]c1cc(-c2cc(F)c(F)c(F)c2)c(CBr)c(-c2c(CBr)c(-c3cc(F)c(F)c(F)c3)cc([R])c2OC)c1OC</smiles>

$(R)-21: \mathrm{R}={ }^{t} \mathrm{Bu}, 78 \%$ yield $(R)-22: \mathrm{R}=\mathrm{H}, 94 \%$ yield

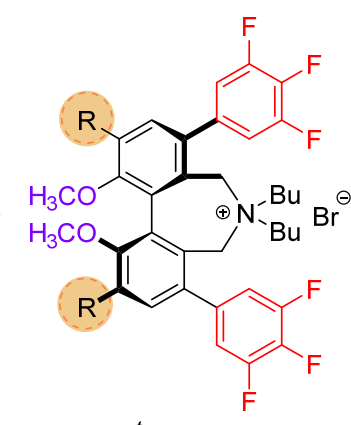

$(R)-23: \mathrm{R}={ }^{t} \mathrm{Bu}, 80 \%$ yield $(R)-24: \mathrm{R}=\mathrm{H}, 81 \%$ yield

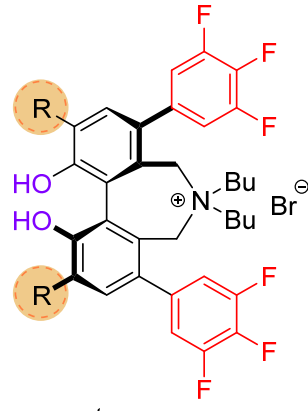

$(R)-25: \mathrm{R}={ }^{t} \mathrm{Bu}, 57 \%$ yield $(R)-26: \mathrm{R}=\mathrm{H}, 50 \%$ yield

图式 4 催化剂 $(R)-23,(R)-24,(R)-25$ 和 $(R)-26$ 的合成路线

Scheme 4 Synthetic route for catalysts $(R)-23,(R)-24,(R)-25$ and $(R)-26$

选择性进行了研究. 以 $N$-二苯基甲亚基甘氨酸叔丁酯 29 和溴化茮作为底物合成甘氨酸衍生物 $\mathbf{3 0}$, 采用已优 化的催化条件 ${ }^{[34-35]}: N$-二苯基甲亚基甘氨酸叔丁酯(29) (1.0 equiv.), 溴化茮(3.0 equiv.), $1 \mathrm{~mol} \%$ 的相转移催化剂,
在甲苯与 $\mathrm{KOH}(50 \%$ aq.)(体积比为 $3: 1)$ 溶液中, 于 $0{ }^{\circ} \mathrm{C}$ 及氩气保护下进行催化不对称烷基化. 所得的 8 个 联苯类相转移催化剂和早期开发的改良 Maruoka 相转 移催化剂 $(S)-27$ 和 $(S)-28$ 的催化结果列于表 1 中. 
表 1 相转移催化甘氨酸衍生物 29 不对称烷基化反应的对映 选择性

Table 1 Catalytic enantioselective phase-transfer alkylation of glycine derivative $\mathbf{2 9}$

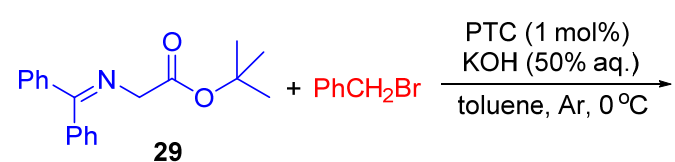<smiles>CC(C)(C)OC(=O)C(Cc1ccccc1)N=C(c1ccccc1)c1ccccc1</smiles>

30

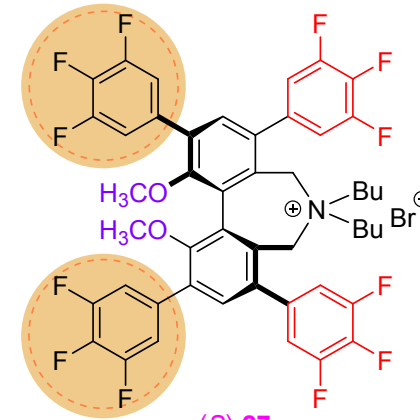

(S)-27

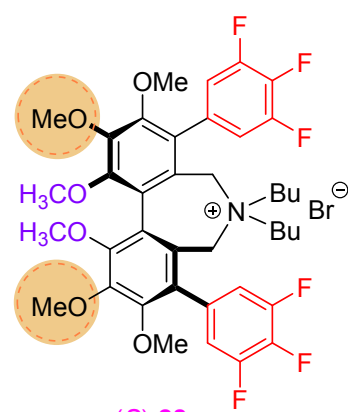

(S)-28

\begin{tabular}{ccccc}
\hline Entry & Catalyst & Time/h & Yield $^{a} \%$ & $e e / \%$ (config.) \\
\hline 1 & $(S)-\mathbf{1 3}$ & 30 & 77 & $81(R)$ \\
2 & $(S)-\mathbf{1 4}$ & 9 & 97 & $93(R)$ \\
3 & $(S)-\mathbf{1 5}$ & 36 & 70 & $61(R)$ \\
4 & $(S)-\mathbf{1 6}$ & 8 & 95 & $91(R)$ \\
5 & $(R)-\mathbf{2 3}$ & 21 & 92 & $76(S)$ \\
6 & $(R)-\mathbf{2 4}$ & 7 & 97 & $96(S)$ \\
7 & $(R)-\mathbf{2 5}$ & 22 & 86 & $64(S)$ \\
8 & $(R)-\mathbf{2 6}$ & 4 & 97 & $91(S)$ \\
9 & $(S)-\mathbf{2 7}$ & 4 & 94 & $86(R)$ \\
10 & $(S)-\mathbf{2 8}$ & 6 & 95 & $98(R)$ \\
\hline
\end{tabular}

${ }^{a}{ }^{1} \mathrm{H}$ NMR yield was determined by using 4-nitroacetophenone as an internal standard.

由表 1 可知, 在 $0{ }^{\circ} \mathrm{C}$ 的两相溶剂中, 联苯类相转移 催化剂能够有效地催化 $N$-二苯基甲亚基甘氨酸叔丁酯 (29)的苄基化反应，反应收率为 $70 \% \sim 97 \%$, ee 值为 $61 \% \sim 98 \%$. 对该系列联苯类相转移催化剂进行构效关 系研究发现: (1) 2 位和 $2^{\prime}$ 位的羟基进行甲基化后, 产率 变化不大, ee 值略微提高. (2) 3 位和 $3^{\prime}$ 位的取代基对反 应活性及选择性影响较大. 当 3 位和 3 '位为氢或小位阻 取代基甲氧基时, 催化活性及选择性都较高, 产率为 $95 \% \sim 97 \%$, ee 值为 91\% 98\% (Entries 2, 4, 6, 8, 10). 当 取代基为 3,4,5-三氟苯基取代基时，产率为 $94 \%, e e$ 值为 $86 \%$, 反应活性及选择性都有所下降(Entry 9). 当 3 位和 3'位引入叔丁基大位阻基团时, 催化剂的反应活性及选 择性有较大幅度的降低(Entries 1, 3, 5, 7). (3) 5 位和 $5^{\prime}$ 位 的取代基对该相转移催化剂的反应活性和选择性起到
非常关键的影响作用. 该位置的取代基一方面协助催化 剂母体骨架构建手性催化空腔，另一方面对母体骨架的 电子分布起到重要的影响. 基于经典 Maruoka 催化剂和 简化的 Maruoka 催化剂(联萗骨架的相转移催化剂)催化 不对称烷基化的研究结果 ${ }^{[35,58]}, 3,4,5$-三氟苯基和 3,5-双 (三氟甲基)苯基有利于反应的选择性，因此我们在联苯 类相转移催化剂的 5 位和 $5^{\prime}$ 位引入 $3,4,5$-三氟苯基和 3,5 双(三氟甲基)苯基. 经过结构改造后，含有两类取代基 的催化剂都显示出较好的反应活性和选择性(Entries 2, $4,6,8,10), 3,4,5$-三氟苯基构建的手性空腔有利于对映 选择性的提高.

\section{2 结论}

综上所述, 本工作以廉价易得的 $(R)-\alpha$-甲氧基苯乙 酸为拆分剂, 对具有轴手性的联苯衍生物的刚性骨架进 行了有效的化学拆分, 为联苯衍生物的拆分提供了更有 效更具实用性的方法. 基于拆分的光学纯的轴手性联苯 衍生物骨架, 设计合成了 8 个联苯类相转移催化剂, 其 中 7 个为新催化剂, 利用核磁共振、高分辨质谱和手性 HPLC 对目标化合物进行结构确认. 在该系列联苯类相 转移催化剂的催化下, 对 $N$-二苯基甲亚基甘氨酸叔丁 酯进行不对称烷基化反应, 研究了该系列催化剂构建手 性甘氨酸衍生物的反应活性及选择性. 构效关系研究发 现，催化剂联苯骨架 2 位和 $2^{\prime}$ 位羟基的甲基化有利于催 化剂的催化效率, 而 3 位和 $3^{\prime}$ 位为氢或引入甲氧基, 有 利于高对映选择性甘氨酸衍生物的构建和合成.

\section{3 实验部分}

\section{1 仪器与试剂}

${ }^{1} \mathrm{H}$ NMR 和 ${ }^{13} \mathrm{C}$ NMR 使用 Bruker AVANCE III 400 $\mathrm{MHz} 、 500 \mathrm{MHz}$ 超导核磁共振仪测定, 样品以気代氯仿 $\left(\mathrm{CDCl}_{3}\right)$ 、甲醇 $\left(\mathrm{MeOD}-d_{4}\right)$ 或丙酮 (acetone- $\left.d_{6}\right)$ 为溶剂, 四 甲基硅烷(TMS)为内标; 高分辨质谱使用 ThermoFisher Q-Exactive (ESI) 测定; 比旋光度使用 Anton Paar MCP-500 全自动数字旋光仪测定; 红外光谱使用 Thermo-Filsher Nicolet 6700 光谱仪测定; 佳航 JH30 熔 点仪测定化合物的熔点. 化合物的对映选择性通过 Shimadzu SPD-16 高效液相色谱仪测定, 手性柱使用 Daicel CHIRALPAK AD-H, OD-H, $4.6 \mathrm{~mm} \times 250 \mathrm{~mm}$. 实 验用的试剂和原料均为分析纯或化学纯.

\section{$3.22,2^{\prime}$-联苯酚衍生物的化学拆分}

\subsubsection{4-溴-2-叔丁基-5-甲基苯酚(2)的合成}

参照文献[59]方法. 室温下，向 2-叔丁基-5-甲基苯 
酚(13.6 mL，80.0 mmol)的甲醇与二氯甲烷的混合溶液 (100 mL, 体积比为 $2: 3)$ 中, 逐滴加入四丁基三溴化铵 $(38.6 \mathrm{~g}, 80.0 \mathrm{mmol})$ 的甲醇与二氯甲烷的混合溶液 $(80$ $\mathrm{mL}$, 体积比为 $2: 3$ ), 搅拌反应 $1 \mathrm{~h}$ 后加水淬灭, 用乙 酸乙酯萃取三次, 合并的有机相经无水硫酸钠干燥, 过 滤, 减压浓缩. 得到的粗产品经硅胶柱层析(乙酸乙酯/ 正己烷, 体积比为 $1: 40 \sim 1: 10$ )分离纯化, 得黄色油 状液体 $\mathbf{2}^{[59]} 19.2 \mathrm{~g}$, 收率 $99 \%$. ${ }^{1} \mathrm{H}$ NMR (400 MHz, $\left.\mathrm{CDCl}_{3}\right) \delta: 7.38(\mathrm{~s}, 1 \mathrm{H}), 6.56(\mathrm{~s}, 1 \mathrm{H}), 4.82(\mathrm{~s}, 1 \mathrm{H}), 2.30(\mathrm{~s}$, $3 \mathrm{H}), 1.39$ (s, 9H); ${ }^{13} \mathrm{C}$ NMR $\left(100 \mathrm{MHz}, \mathrm{CDCl}_{3}\right) \delta: 153.4$, 136.3, 136.0, 130.9, 119.0, 115.5, 34.5, 29.6, 22.2.

3.2.2 5, ' ' -二溴-3,3'-二叔丁基-6,6'-二甲基-2,2'-联苯 酚(3)的合成

参照文献[59]方法. 室温下, 将化合物 2 (243.1 mg, $1.0 \mathrm{mmol}) 、$ 氯化亚铜 $(9.90 \mathrm{mg}, 0.1 \mathrm{mmol})$ 和四甲基乙二 胺 $(22 \mu \mathrm{L}, 0.15 \mathrm{mmol})$ 加入二氯甲烷 $(2 \mathrm{~mL})$ 中, 剧烈搅拌 反应过夜后, 加水淬灭. 体系经二氯甲烷萃取三次, 合 并后的有机相用无水硫酸钠干燥, 过滤, 减压浓缩. 得 到的粗产品经硅胶柱层析(洗脱剂为正己烷)分离纯化, 得白色固体 $\mathbf{3}^{[59]} 123.5 \mathrm{mg}$, 收率 51\%. m.p. 203 205 ${ }^{\circ} \mathrm{C}$; ${ }^{1} \mathrm{H}$ NMR (400 MHz, $\left.\mathrm{CDCl}_{3}\right) \delta: 7.55$ (s, 2H), 4.88 (s, 2H), $2.00(\mathrm{~s}, 6 \mathrm{H}), 1.40(\mathrm{~s}, 18 \mathrm{H}) ;{ }^{13} \mathrm{C} \mathrm{NMR}\left(100 \mathrm{MHz}, \mathrm{CDCl}_{3}\right) \delta$ : 151.8, 136.8, 135.5, 132.0, 121.8, 116.4, 35.0, 29.4, 19.9.

\section{$3.2 .3(R)-\alpha$-甲氧基苯乙酰氯的合成}

参照文献 $[60]$ 方法. 在 $0{ }^{\circ} \mathrm{C}$ 和氩气保护的条件下, 向 $(R)-\alpha$-甲氧基苯乙酸(7.98 g, $48 \mathrm{mmol}$ )的无水二氯甲烷 $(70 \mathrm{~mL})$ 溶液中缓慢滴加 $2 \mathrm{~mol} / \mathrm{L}$ 草酰氯 $(24 \mathrm{~mL}, 48$ $\mathrm{mmol}$ )和无水的 $N, N$-二甲基甲酰胺 $(800 \mu \mathrm{L})$, 在 $0{ }^{\circ} \mathrm{C}$ 反 应 $15 \mathrm{~min}$ 后置于室温反应 $6 \mathrm{~h}$. 浓缩, 抽干 $15 \mathrm{~min}$ 得 $(R)-\alpha$-甲氧基苯基乙酰氯，直接用于下一步反应.

\section{2 .4 化合物 $(R, S)-4$ 和 $(R, R, R)-5$ 的合成}

参照文献[61]方法. 在 $0{ }^{\circ} \mathrm{C}$ 和氩气保护的条件下, 向氢化钠 $(1.44 \mathrm{~g}, 36 \mathrm{mmol})$ 的干燥四氢呋喃 $(80 \mathrm{~mL})$ 混合 液中，缓慢滴入化合物 $3(5.81 \mathrm{~g}, 12 \mathrm{mmol})$ 的干燥四氢 呋喃 $(50 \mathrm{~mL})$ 溶液, 滴加完毕后, 反应混合物在常温下继 续摚拌反应 $3 \mathrm{~h}$. 将现制的 $(R)-\alpha$-甲氧基苯乙酰氯(8.86 g, $48 \mathrm{mmol}$ )逐滴加入上述反应体系中, 剧烈搅拌反应 $1 \mathrm{~h}$, 将反应体系缓慢倒入冰水中，二氯甲烷萃取三次，合并 后的有机相, 用无水硫酸钠干燥, 过滤, 减压浓缩. 得 到的粗产品经硅胶柱层析(乙酸乙酯/二氯甲烷/正己烷, 体积比 $1: 1: 90 \sim 1: 1: 50$ )分离纯化, 得白色固体目 标物 $(R, S)-\mathbf{4}$ 和 $(R, R, R)-\mathbf{5}$.

(S)-3',5-二溴-3,5'-二叔丁基-6'-羟基-2',6-二甲基-
[1,1'-联苯基]-2-(2-甲氧基- $(R)$-2-苯基乙酸酯)酯 $[(R, S)-4]$ : $3.72 \mathrm{~g}$, 收率 $49 \%$. 经重结晶(二氯甲烷/正己烷, 体积比 为 $1: 2$ )后, $e e>99 \%$. 白色固体, m.p. $107 \sim 108{ }^{\circ} \mathrm{C}$; $[\alpha]_{\mathrm{D}}^{25}$ +6 (c 0.55, $\left.\mathrm{CHCl}_{3}\right) ;{ }^{1} \mathrm{H}$ NMR $\left(400 \mathrm{MHz}, \mathrm{CDCl}_{3}\right) \delta: 7.60$ $(\mathrm{s}, 1 \mathrm{H}), 7.41(\mathrm{~s}, 1 \mathrm{H}), 7.33 \sim 7.35(\mathrm{~m}, 3 \mathrm{H}), 7.25 \sim 7.23(\mathrm{~m}$, 1H), $5.05(\mathrm{~s}, 1 \mathrm{H}), 4.17(\mathrm{~s}, 1 \mathrm{H}), 3.02(\mathrm{~s}, 3 \mathrm{H}), 2.01(\mathrm{~s}, 3 \mathrm{H})$, 1.94 (s, 3H), 1.35 (s, 9H), 0.97 (s, 9H); ${ }^{13} \mathrm{C}$ NMR (100 $\left.\mathrm{MHz}, \mathrm{CDCl}_{3}\right) \delta: 169.6,151.7,147.7,142.2,138.0,137.1$, $134.7,134.0,131.9,131.5,130.7,129.2,128.8,127.8$, 126.6, 123.4, 116.2, 82.9, 57.6, 34.9, 34.4, 29.6, 29.4, 20.0, 19.8; IR (KBr) v: 3468, 3068, 3033, 3007, 2962, 2914, 2876, 2828, 1764, 1553, 1489, 1457, 1396, 1364, 1277, 1261, 1207, 1146, 1079, 1028, 983, 909, 871, 829, 765, $749,698,650,592,553,508 \mathrm{~cm}^{-1}$; HRMS (ESI) calcd for $\mathrm{C}_{31} \mathrm{H}_{36} \mathrm{Br}_{2} \mathrm{O}_{4} \mathrm{Na}[\mathrm{M}+\mathrm{Na}]^{+}: 653.0878$, found 653.0879 .

(R)-5,5'-二溴-3,3'-二叔丁基-6,6'-二甲基-[1, $1^{\prime}$ - 联苯 基]-(2R,2' $R$ )- $2,2^{\prime}$-双(2-甲氧基-2-苯基乙酸酯)酯 $[(R, R, R)$ $5]: 2.99 \mathrm{~g}$, 收率 $30 \%$. 经重结晶(二氯甲烷/正己烷，体积 比为 $1: 3$ )后, $e e>99 \%$. 白色固体, m.p. 199 200 ${ }^{\circ} \mathrm{C}$; $[\alpha]_{\mathrm{D}}^{25}-35.7\left(c 0.1, \mathrm{CHCl}_{3}\right) ;{ }^{1} \mathrm{H}$ NMR $\left(400 \mathrm{MHz}, \mathrm{CDCl}_{3}\right) \delta$ : $7.60(\mathrm{~s}, 2 \mathrm{H}), 7.44 \sim 7.34(\mathrm{~m}, 10 \mathrm{H}), 3.98(\mathrm{~s}, 2 \mathrm{H}), 2.99(\mathrm{~s}$, $6 \mathrm{H}), 2.14(\mathrm{~s}, 6 \mathrm{H}), 0.94(\mathrm{~s}, 18 \mathrm{H}) ;{ }^{13} \mathrm{C}$ NMR (100 MHz, $\left.\mathrm{CDCl}_{3}\right) \delta: 168.0,146.9,141.6,138.0,135.0,132.3,131.3$, $129.1,128.6,127.8,123.6,82.2,58.0,34.2,29.9,20.4$; IR (KBr) v: 3423, 3058, 3004, 2962, 2917, 2869, 2828, 1768, 1543, 1489, 1454, 1441, 1399, 1367, 1354, 1322, 1281, $1258,1213,1143,1111,1085,1028,992,964,909,887$, 823, 762, 749, 733, 695, 643, 573, 554, $518 \mathrm{~cm}^{-1}$; HRMS (ESI) calcd for $\mathrm{C}_{40} \mathrm{H}_{44} \mathrm{Br}_{2} \mathrm{O}_{6} \mathrm{Na}[\mathrm{M}+\mathrm{Na}]^{+}$: 803.1376, found 803.1381 .

3.2 .5 化合物 $(S)-3 、(R)-3$ 的合成

参照文献[62]方法. 在 $0{ }^{\circ} \mathrm{C}$ 及氩气保护的条件下, 向 $(R, S)$-4 (556.5 mg, $0.88 \mathrm{mmol})$ 的无水四氢呋喃 $(2 \mathrm{~mL})$ 溶液中，缓慢滴加 $1 \mathrm{~mol} / \mathrm{L}$ 四氢铝锂 $(1.8 \mathrm{~mL}, 1.76$ $\mathrm{mmol}$ ), 反应 $1 \mathrm{~h}$, 将反应体系缓慢倒入冰水混合物中, 二氯甲烷萃取三次，合并后的有机相，用无水硫酸钠干 燥, 过滤, 减压浓缩. 得到的粗产品经硅胶柱层析(正己 烷)分离纯化, 得 $409.3 \mathrm{mg}(S)-5,5^{\prime}$-二溴-3,3'-二叔丁基6,6'-二甲基-2,2'-联苯酚 $[(S)-3]$ ，收率 96\%。白色固体， m.p. $203 \sim 205{ }^{\circ} \mathrm{C} ;{ }^{1} \mathrm{H}$ NMR $\left(400 \mathrm{MHz}, \mathrm{CDCl}_{3}\right) \delta$ : 7.54 (s, 2H), 4.87 (s, 2H), 1.99 (s, 6H), 1.39 (s, 18H); ${ }^{13} \mathrm{C}$ NMR $\left(100 \mathrm{MHz}, \mathrm{CDCl}_{3}\right) \delta: 151.8,136.8,135.5,132.0,121.7$, 116.4, 35.0, 29.4, 19.9 .

( $R$ )-5,5'-二溴-3,3'-二叔丁基-6,6'-二甲基-2,2'-联苯酚 
$(R)-3$ : 合成步骤与 $(S)-3$ 相同, $669.3 \mathrm{mg}$, 产率 $96 \%$. 白色 固体, m.p. 203 205 ${ }^{\circ} \mathrm{C} ;{ }^{1} \mathrm{H}$ NMR (400 MHz, $\left.\mathrm{CDCl}_{3}\right) \delta$ : 7.54 (s, 2H), 4.87 (s, 2H), 1.99 (s, 6H), 1.39 (s, 18H); ${ }^{13} \mathrm{C}$ NMR $\left(100 \mathrm{MHz}, \mathrm{CDCl}_{3}\right) \delta: 151.8,136.8,135.5,132.0$, $121.7,116.4,35.0,29.4,19.9$.

\subsection{6 化合物 $(S)-6$ 和 $(R)-6$ 的合成}

参照文献[59]方法. 在 $0{ }^{\circ} \mathrm{C}$ 及氩气保护的条件下, 向 $(S)$-3 (377.7 mg, $0.78 \mathrm{mmol})$ 的甲苯 $(4 \mathrm{~mL})$ 溶液中缓慢 滴加三氟甲磺酸 $(158.0 \mu \mathrm{L}, 1.79 \mathrm{mmol})$, 继续搅拌 15 $\min$. 将反应体系倒入冰水混合物中, 用二氯甲烷萃取 三次, 合并后的有机相用无水硫酸钠干燥, 过滤, 减压 浓缩. 得到的粗产品经硅胶柱层析 (乙酸乙酯/正己烷, $V: V=1: 10)$ 分离纯化, 得 $272.8 \mathrm{mg}(S)-5,5^{\prime}$-二溴-6,6'二甲基-2,2'-联苯酚 $[(S)-6]$, 收率 $94 \%, e e>99 \%$. 白色固 体. m.p. 204 206 ${ }^{\circ} \mathrm{C} ;{ }^{1} \mathrm{H}$ NMR (400 MHz, $\left.\mathrm{CDCl}_{3}\right) \delta$ : 7.54 (d, $J=8.4 \mathrm{~Hz}, 2 \mathrm{H}), 6.81$ (d, $J=8.8 \mathrm{~Hz}, 2 \mathrm{H}), 4.76$ (s, 2H), 2.07 (s, 6H); ${ }^{13} \mathrm{C}$ NMR (100 MHz, $\left.\mathrm{CDCl}_{3}\right) \delta: 153.1$, 138.3, 134.3, 121.7, 116.6, 115.3, 20.3; HPLC: Diacel Chiralpak AD-H (5 $\mu \mathrm{m}, 4.6 \mathrm{~mm} \times 250 \mathrm{~mm}), V$ (hexane)/ $V(\mathrm{EtOH})=20 / 1$, flow rate $=1.0 \mathrm{~mL} / \mathrm{min}, \lambda=290 \mathrm{~nm}$, retention time: $25.1 \mathrm{~min}[(S)$-enantiomer $], 55.5 \mathrm{~min}[(R)$ enantiomer].

$(R)-5,5^{\prime}$-二溴-6,6'-二甲基- $2,2^{\prime}$-联苯酚 $[(R)-6]$ : 合成步 骤与 $(S)-6$ 相同, 产率 94\%, ee $>99 \%$. 白色固体, m.p. 204 206 ${ }^{\circ} \mathrm{C} ;{ }^{1} \mathrm{H}$ NMR (400 MHz, $\left.\mathrm{CDCl}_{3}\right) \delta: 7.54$ (d, $J=$ $8.4 \mathrm{~Hz}, 2 \mathrm{H}), 6.81(\mathrm{~d}, J=8.8 \mathrm{~Hz}, 2 \mathrm{H}), 4.76(\mathrm{~s}, 2 \mathrm{H}), 2.07$ (s, $6 \mathrm{H}) ;{ }^{13} \mathrm{C}$ NMR (100 MHz, $\left.\mathrm{CDCl}_{3}\right) \delta: 153.1,138.3,134.3$, 121.7, 116.6, 115.3, 20.3; HPLC: Diacel Chiralpak AD-H $(5 \mu \mathrm{m}, 4.6 \mathrm{~mm} \times 250 \mathrm{~mm}), V($ hexane $) / V(\mathrm{EtOH})=20 / 1$, flow rate $=1.0 \mathrm{~mL} / \mathrm{min}, \lambda=290 \mathrm{~nm}$, retention time: 24.6 $\min [(S)$-enantiomer], $54.6 \mathrm{~min}[(R)$-enantiomer].

\section{3 相转移催化剂的合成}

参照文献[63]方法. 在氩气保护的条件下, 将 $(S)-3$ (368.0 mg, $0.76 \mathrm{mmol}$ )、3,5-双(三氟甲基)苯喼酸(469.4 $\mathrm{mg}, 1.82 \mathrm{mmol}) 、$ 四三苯基膦钯 $(87.8 \mathrm{mg}, 0.076 \mathrm{mmol})$ 和 碳酸钠 $(322.2 \mathrm{mg}, 3.04 \mathrm{mmol})$ 加入到乙二醇二甲醚(3 $\mathrm{mL})$ 和水 $(1.5 \mathrm{~mL})$ 的混合溶液中, 在 $90{ }^{\circ} \mathrm{C}$ 下反应过夜. 将体系冷却至室温后, 缓慢加入饱和氯化铵溶液 (10 $\mathrm{mL}$ ), 用二氯甲烷萃取三次, 合并后的有机相用无水硫 酸钠干燥, 过滤, 减压浓缩. 得到的粗产品经硅胶柱层 析(正己烷为洗脱剂)分离纯化, 得 $553.1 \mathrm{mg}(S)-3,3^{\prime}-$ 二 叔丁基-6,6'-二甲基-5, ' '二 二 [3,5-双 (三氟甲基)苯基]-2,2'联苯酚 $[(S)-7]$, 收率 97\%. 白色固体, m.p. $71 \sim 73{ }^{\circ} \mathrm{C}$;
$[\alpha]_{\mathrm{D}}^{25}-15.0\left(c\right.$ 0.78, $\left.\mathrm{CHCl}_{3}\right) ;{ }^{1} \mathrm{H} \mathrm{NMR}\left(500 \mathrm{MHz}, \mathrm{CDCl}_{3}\right)$ $\delta: 7.87(\mathrm{~s}, 2 \mathrm{H}), 7.80(\mathrm{~s}, 4 \mathrm{H}), 7.24(\mathrm{~s}, 2 \mathrm{H}), 5.19(\mathrm{~s}, 2 \mathrm{H})$, $1.86(\mathrm{~s}, 6 \mathrm{H}), 1.45(\mathrm{~s}, 18 \mathrm{H}) ;{ }^{13} \mathrm{C} \mathrm{NMR}\left(125 \mathrm{MHz}, \mathrm{CDCl}_{3}\right) \delta$ : $152.8,144.3,135.2,133.4,132.2,131.7\left(\mathrm{q}, J_{\mathrm{C}-\mathrm{F}}=33.0\right.$ $\mathrm{Hz}), 129.86 \sim 129.84(\mathrm{~m}), 129.7,123.5\left(\mathrm{q}, J_{\mathrm{C}-\mathrm{F}}=271.1\right.$ $\mathrm{Hz}), 121.2,120.8 \sim 120.7$ (m), 35.0, 29.5, 17.3; IR (KBr) v: 3519, 3427, 2965, 2921, 2869, 1620, 1463, 1444, 1390, $1370,1325,1281,1178,1140,1108,1085,1024,1005$, 903, 848, 730, 711, 688, 672, 631, 589, $457 \mathrm{~cm}^{-1}$; HRMS (ESI) calcd for $\mathrm{C}_{38} \mathrm{H}_{33} \mathrm{~F}_{12} \mathrm{O}_{2}[\mathrm{M}-\mathrm{H}]^{-}:$749.2294, found 749.2296 .

(S)-6,6'-二甲基-5,5'-二[3,5-双 (三氟甲基)苯基]-2,2'联苯酚 $[(S)-8]$ : 合成步骤与 $(S)-7$ 相同, 产率 94\%. 白色 固体, m.p. $180 \sim 183{ }^{\circ} \mathrm{C}$; $[\alpha]_{\mathrm{D}}^{25}+43.7\left(c 0.10, \mathrm{CHCl}_{3}\right) ;{ }^{1} \mathrm{H}$ NMR (400 MHz, $\left.\mathrm{CDCl}_{3}\right) \delta: 7.88(\mathrm{~s}, 2 \mathrm{H}), 7.81(\mathrm{~s}, 4 \mathrm{H}), 7.28$ (d, $J=8.4 \mathrm{~Hz}, 2 \mathrm{H}), 7.04(\mathrm{~d}, J=8.4 \mathrm{~Hz}, 2 \mathrm{H}), 5.17$ (s, 2H), $1.97(\mathrm{~s}, 6 \mathrm{H}) ;{ }^{13} \mathrm{C} \mathrm{NMR}\left(100 \mathrm{MHz}, \mathrm{CDCl}_{3}\right) \delta: 154.1,143.7$, $136.3,133.0,132.2,131.8\left(\mathrm{q}, J_{\mathrm{C}-\mathrm{F}}=33.1 \mathrm{~Hz}\right), 129.84 \sim$ $129.82(\mathrm{~m}), 123.5\left(\mathrm{q}, J_{\mathrm{C}-\mathrm{F}}=271.1 \mathrm{~Hz}\right), 121.0,121.96 \sim$ 120.90 (m), 114.1, 17.7; IR (KBr) v: 3478, 3385, 1616, $1582,1485,1463,1376,1338,1277,1258,1178,1133$, 1104, 1047, 995, 890, 841, 816, 707, 678, $617 \mathrm{~cm}^{-1}$; HRMS (ESI) calcd for $\mathrm{C}_{30} \mathrm{H}_{17} \mathrm{~F}_{12} \mathrm{O}_{2}[\mathrm{M}-\mathrm{H}]^{-}:$637.1042, found 637.1046 .

( $R$ )- $3,3^{\prime}$-二叔丁基-6,6'-二甲基-5,5'-双 $(3,4,5$-三氟苯 基)-2,2'-联苯酚 $[(R)-17]$ : 合成步骤与 $(S)-7$ 相同，产率 $93 \%$. 白色固体, m.p. $223 \sim 225{ }^{\circ} \mathrm{C} ;[\alpha]_{\mathrm{D}}^{25}+20.9$ (c 0.22 , $\left.\mathrm{CHCl}_{3}\right) ;{ }^{1} \mathrm{H} \mathrm{NMR}\left(400 \mathrm{MHz}, \mathrm{CDCl}_{3}\right) \delta: 7.20(\mathrm{~s}, 2 \mathrm{H}), 6.96$ (dd, $J=8.4,6.4 \mathrm{~Hz}, 4 \mathrm{H}), 5.12$ (s, 2H), $1.86(\mathrm{~s}, 6 \mathrm{H}), 1.44$ (s, $18 \mathrm{H}) ;{ }^{13} \mathrm{C}$ NMR (100 MHz, $\left.\mathrm{CDCl}_{3}\right) \delta: 152.4,150.96$ (ddd, $\left.J_{\mathrm{C}-\mathrm{F}}=248.5,9.8,4.3 \mathrm{~Hz}\right), 138.9\left(\mathrm{dt}, J_{\mathrm{C}-\mathrm{F}}=249.5,15.3\right.$ $\mathrm{Hz}), 138.2 \sim 138.4(\mathrm{~m}), 134.8,133.2,132.3,129.4,121.1$, $113.9\left(\mathrm{dd}, J_{\mathrm{C}-\mathrm{F}}=15.2,5.6 \mathrm{~Hz}\right), 34.9,29.6,17.2$; IR $(\mathrm{KBr})$ $v: 3513,3443,2959,2917,2856,1630,1620,1575,1530$, $1470,1434,1358,1322,1271,1239,1197,1178,1044$, 903, 858, 768, 701, 647, 598, $467 \mathrm{~cm}^{-1}$; HRMS (ESI) calcd for $\mathrm{C}_{34} \mathrm{H}_{31} \mathrm{~F}_{6} \mathrm{O}_{2}[\mathrm{M}-\mathrm{H}]^{-}:$585.2233, found 585.2235.

( $R$ )- $6,6^{\prime}$-二甲基-5,5'-双(3,4,5-三氟苯基)-2,2'-联苯酚 [(R)-18]: 合成步骤与 $(S)-7$ 相同, 产率 95\%. 白色固体, m.p. $228 \sim 229{ }^{\circ} \mathrm{C}$; $[\alpha]_{\mathrm{D}}^{25}-47.6\left(c 0.45, \mathrm{CHCl}_{3}\right)$; ${ }^{1} \mathrm{H}$ NMR $\left(400 \mathrm{MHz}\right.$, acetone- $\left.d_{6}\right) \delta: 7.86(\mathrm{~s}, 2 \mathrm{H}), 7.12 \sim 7.16(\mathrm{~m}$, $6 \mathrm{H}), 6.91(\mathrm{~d}, J=8.4 \mathrm{~Hz}, 2 \mathrm{H}), 1.96(\mathrm{~s}, 6 \mathrm{H}) ;{ }^{13} \mathrm{C}$ NMR $(100$ $\mathrm{MHz}$, acetone- $\left.d_{6}\right) \delta: 155.8,151.4\left(\mathrm{ddd}, J_{\mathrm{C}-\mathrm{F}}=246.2,9.5\right.$, $4.2 \mathrm{~Hz}), 140.1 \sim 140.3(\mathrm{~m}), 139.1\left(\mathrm{dt}, J_{\mathrm{C}-\mathrm{F}}=246.5,15.3\right.$ 
$\mathrm{Hz}), 136.5,132.0,130.8,125.2,114.7\left(\mathrm{dd}, J_{\mathrm{C}-\mathrm{F}}=15.3,5.4\right.$ $\mathrm{Hz}), 114.1,17.9$; IR (KBr) v: 3462, 3366, 3087, 2965, 2911, 2850, 1614, 1582, 1527, 1473, 1422, 1402, 1358, 1284, 1236, 1188, 1153, 1092, 1056, 1018, 999, 871, 855, $819,755,711,627,582,547,499 \mathrm{~cm}^{-1}$; HRMS (ESI) calcd for $\mathrm{C}_{26} \mathrm{H}_{15} \mathrm{~F}_{6} \mathrm{O}_{2}[\mathrm{M}-\mathrm{H}]^{-}: 473.0981$, found 473.0985.

(S)-3,3'-二叔丁基-2,2'-二甲氧基-6,6'-二甲基-5,5'-二 [3,5-双 (三氟甲基)苯基]联苯 [(S)-9]: 参照文献[35]方法. 向化合物 $(S)-7$ (510.4 mg, $0.68 \mathrm{mmol})$ 和碳酸钾(939.7 $\mathrm{mg}, 6.8 \mathrm{mmol}$ )的丙酩混合液 $(6.0 \mathrm{~mL})$ 中缓慢滴加碘甲烷 $(846 \mu \mathrm{L}, 13.6 \mathrm{mmol})$, 反应回流过夜. 将反应体系冷却 至室温后, 加水终止反应, 用二氯甲烷萃取三次, 合并 后的有机相, 用无水硫酸钠干燥, 过滤, 减压浓缩. 得 到的粗产品经硅胶柱层析 (乙酸乙酯/正己烷, 体积比 $1: 100)$ 分离纯化, 得 $524.2 \mathrm{mg}(S)-9^{[35]}$, 收率 $99 \%$. 白色 固体, m.p. $139 \sim 141{ }^{\circ} \mathrm{C} ;[\alpha]_{\mathrm{D}}^{25}+11.7\left(\mathrm{c} 0.10, \mathrm{CHCl}_{3}\right) ;{ }^{1} \mathrm{H}$ NMR (400 MHz, $\left.\mathrm{CDCl}_{3}\right) \delta: 7.87$ (s, 2H), $7.79(\mathrm{~s}, 4 \mathrm{H}), 7.20$ (s, 2H), 3.31 (s, 6H), 1.94 (s, 9H), 1.43 (s, 18H); ${ }^{13} \mathrm{C}$ NMR $\left(100 \mathrm{MHz}, \mathrm{CDCl}_{3}\right) \delta: 157.9,145.0,141.3,134.6,133.7$, $132.9,131.6\left(\mathrm{q}, J_{\mathrm{C}-\mathrm{F}}=33.1 \mathrm{~Hz}\right), 129.8 \sim 129.7(\mathrm{~m}), 128.0$, $123.6\left(\mathrm{q}, J_{\mathrm{C}-\mathrm{F}}=271.1 \mathrm{~Hz}\right), 120.8 \sim 120.7(\mathrm{~m}), 59.6,35.1$, 30.8, 18.1; IR (KBr) v: 3430, 3010, 2965, 2933, 2866, 1617, 1492, 1463, 1393, 1354, 1274, 1178, 1117, 1101, 1069, 1047, 967, 900, 890, 842, 717, 672, $576 \mathrm{~cm}^{-1}$. HRMS (ESI) calcd for $\mathrm{C}_{36} \mathrm{H}_{35} \mathrm{~F}_{6} \mathrm{O}_{2}[\mathrm{M}-\mathrm{H}]^{-}:$613.2541, found 613.2537

(S)-2,2'-二甲氧基-6,6'-二甲基-5,5'-二[3,5-双 (三氟甲 基)苯基]联苯 $[(S)-10]$ : 合成步骤与 $(S)-9$ 相同, 白色固 体, 产率 99\%. m.p. 39 41 ${ }^{\circ} \mathrm{C} ;[\alpha]_{\mathrm{D}}^{25}+2.8$ (c 0.54 , $\left.\mathrm{CHCl}_{3}\right) ;{ }^{1} \mathrm{H} \mathrm{NMR}\left(400 \mathrm{MHz}, \mathrm{CDCl}_{3}\right) \delta: 7.76(\mathrm{~s}, 2 \mathrm{H}), 7.74$ (s, 4H), 7.16 (d, $J=8.4 \mathrm{~Hz}, 2 \mathrm{H}), 6.87$ (d, $J=8.8 \mathrm{~Hz}, 2 \mathrm{H})$, $3.71(\mathrm{~s}, 6 \mathrm{H}), 1.79(\mathrm{~s}, 6 \mathrm{H}) ;{ }^{13} \mathrm{C} \mathrm{NMR}\left(100 \mathrm{MHz}, \mathrm{CDCl}_{3}\right) \delta$ : $157.2,144.5,135.5,132.25,131.5\left(\mathrm{q}, J_{\mathrm{C}-\mathrm{F}}=33.1 \mathrm{~Hz}\right)$, $130.1,129.96 \sim 129.93(\mathrm{~m}), 127.2,123.6\left(\mathrm{q}, J_{\mathrm{C}-\mathrm{F}}=216.9\right.$ $\mathrm{Hz}), 120.6 \sim 120.4(\mathrm{~m}), 108.8,56.08,17.6$; IR (KBr) $v$ : 3443, 2962, 2840, 1623, 1588, 1495, 1460, 1383, 1277, $1175,1127,1082,1050,900,848,810,707,685,582$ $\mathrm{cm}^{-1}$. HRMS (ESI) calcd for $\mathrm{C}_{32} \mathrm{H}_{21} \mathrm{~F}_{12} \mathrm{O}_{2}[\mathrm{M}-\mathrm{H}]^{-}$: 665.1350, found 665.1348

(R)-3,3'-二叔丁基-2,2'-二甲氧基-6,6'-二甲基-5,5'-双 (3,4,5-三氟苯基)联苯 $[(R)-19]$ : 合成步骤与 $(S)-9$ 相同, 白色固体, 产率 99\%. m.p. $177 \sim 179{ }^{\circ} \mathrm{C} ;[\alpha]_{\mathrm{D}}^{25}-17.3$ (c $\left.0.10, \mathrm{CHCl}_{3}\right) ;{ }^{1} \mathrm{H} \mathrm{NMR}\left(400 \mathrm{MHz}, \mathrm{CDCl}_{3}\right) \delta: 7.08(\mathrm{~s}, 2 \mathrm{H})$, $6.86(\mathrm{dd}, J=8,6.8 \mathrm{~Hz}, 4 \mathrm{H}), 3.17$ (s, 6H), 1.85 (s, 6H), 1.31 (s, 18H); ${ }^{13} \mathrm{C}$ NMR (100 MHz, $\left.\mathrm{CDCl}_{3}\right) \delta: 157.6,151.0 \mathrm{ddd}$, $\left.J_{\mathrm{C}-\mathrm{F}}=248.4,9.9,4.1 \mathrm{~Hz}\right), 141.0(\mathrm{~s}), 138.8 \sim 139.0(\mathrm{~m})$, $138.9\left(\mathrm{dt}, J_{\mathrm{C}-\mathrm{F}}=249.2,15.3 \mathrm{~Hz}\right), 134.7,133.7,132.8$, $127.8,113.8\left(\mathrm{dd}, J_{\mathrm{C}-\mathrm{F}}=15.2,5.5 \mathrm{~Hz}\right), 59.4,35.0,30.8$, 18.1; IR (KBr) v: 3439, 2969, 2946, 2914, 2856, 1614, 1521, 1447, 1415, 1396, 1354, 1261, 1239, 1229, 1201, $1165,1069,1037,983,874,855,839,765,723,695,634$, $586 \mathrm{~cm}^{-1}$. HRMS (ESI) calcd for $\mathrm{C}_{36} \mathrm{H}_{35} \mathrm{~F}_{6} \mathrm{O}_{2}[\mathrm{M}-\mathrm{H}]^{-}$: 613.2541, found 613.2537.

(R)-2,2'-二甲氧基-6,6'-二甲基-5, ''-双 (3,4,5-三氟苯 基)联苯 $[(R)-20]$ : 合成步骤与(S)-9 相同, 产率 99\%. 白 色固体, m.p. 56 57 ${ }^{\circ} \mathrm{C} ;{ }^{1} \mathrm{H}$ NMR $\left(400 \mathrm{MHz}, \mathrm{CDCl}_{3}\right) \delta$ : $7.18(\mathrm{~d}, J=8.4 \mathrm{~Hz}, 2 \mathrm{H}), 6.96(\mathrm{t}, J=7.4 \mathrm{~Hz}, 4 \mathrm{H}), 6.89$ (d, $J=8.4 \mathrm{~Hz}, 2 \mathrm{H}), 3.76$ (s, 6H), $1.83(\mathrm{~s}, 6 \mathrm{H}) ;{ }^{13} \mathrm{C}$ NMR $(100$ $\mathrm{MHz}, \mathrm{CDCl}_{3}$ ) $\delta: 156.9,150.86\left(\mathrm{ddd}, J_{\mathrm{C}-\mathrm{F}}=248.3,9.8,4.2\right.$ $\mathrm{Hz}), 138.6\left(\mathrm{dt}, J_{\mathrm{C}-\mathrm{F}}=249.0,15.2 \mathrm{~Hz}\right), 138.4 \sim 138.6(\mathrm{~m})$, $135.4,132.4,129.7,127.0,113.9\left(\mathrm{dd}, J_{\mathrm{C}^{-} \mathrm{F}}=15.3,5.5 \mathrm{~Hz}\right)$, 108.5, 55.9, 17.6.

(S)-6,6'-二澳甲基-3,3'-二叔丁基-2,2'-二甲氧基-5,5'二[3,5-双 (三氟甲基)苯基]联苯 [(S)-11]: 参照文献[35]方 法. 向化合物(S)-9 (420.5 mg, $0.54 \mathrm{mmol})$ 和 $N$-溴代丁二 酰亚胺 $(673.0 \mathrm{mg}, 3.78 \mathrm{mmol})$ 的苯 $(5 \mathrm{~mL})$ 的混合溶液中 加入偶氮二异丁腈 $(26.6 \mathrm{mg}, 30 \mathrm{~mol} \%)$, 在回流状态下 反应 $4 \mathrm{~h}$. 将反应体系冷却至室温, 加入乙醚稀释、过滤. 滤液浓缩后所得的粗产物经硅胶柱层析(乙酸乙酯/正己 烷, $1: 100)$ 分离纯化, 得 $476.9 \mathrm{mg}$ 目标物(S)-11 ${ }^{[35]}$, 收 率 94\%. 黄色固体, m.p. $61 \sim 63{ }^{\circ} \mathrm{C} ;[\alpha]_{\mathrm{D}}^{25}+28.7$ (c 0.10, $\mathrm{CHCl}_{3}$ ); ${ }^{1} \mathrm{H}$ NMR (400 MHz, $\left.\mathrm{CDCl}_{3}\right) \delta: 7.85(\mathrm{~s}, 2 \mathrm{H}), 7.83$ (s, 2H), $7.20(\mathrm{~s}, 2 \mathrm{H}), 4.14$ (d, J=10.8 Hz, 2H), 4.00 (d, $J=10.4 \mathrm{~Hz}, 2 \mathrm{H}), 3.38$ (s, 6H), $1.34(\mathrm{~s}, 18 \mathrm{H}) ;{ }^{13} \mathrm{C} \mathrm{NMR}$ $\left(100 \mathrm{MHz}, \mathrm{CDCl}_{3}\right) \delta: 157.0,144.9,143.2,136.4,133.5$, $131.7\left(\mathrm{q}, J_{\mathrm{C}-\mathrm{F}}=33.4 \mathrm{~Hz}\right), 130.8,130.2,129.81 \sim 129.78$ $(\mathrm{m}), 123.5\left(\mathrm{q}, J_{\mathrm{C}-\mathrm{F}}=271.1 \mathrm{~Hz}\right), 121.6 \sim 121.5(\mathrm{~m}), 60.0$, 35.5, 30.6, 30.1; IR (KBr) v: 3443, 2962, 2914, 2869, 1623, 1466, 1390, 1354, 1277, 1185, 1130, 1050, 900, 845, 733, 711, 682, 621, $576 \mathrm{~cm}^{-1}$; HRMS (ESI) calcd for $\mathrm{C}_{41} \mathrm{H}_{37} \mathrm{Br}_{2} \mathrm{~F}_{12} \mathrm{O}_{4}[\mathrm{M}+\mathrm{HCOOH}-\mathrm{H}]^{-}:$981.0852, found 981.0869.

(S)-6,6'-二溴甲基-2,2'-二甲氧基-5, ''-二 [3,5-双 (三氟 甲基)苯基]联苯 $[(S)-12]$ : 合成步骤与 $(S)-\mathbf{1 1}$ 相同, 产率 96\%. 黄色固体, m.p. $66 \sim 68{ }^{\circ} \mathrm{C}$; $[\alpha]_{\mathrm{D}}^{25}+28$ (c 0.10 , $\left.\mathrm{CHCl}_{3}\right) ;{ }^{1} \mathrm{H} \mathrm{NMR}\left(400 \mathrm{MHz}, \mathrm{CDCl}_{3}\right) \delta$ : $7.94(\mathrm{~s}, 2 \mathrm{H}), 7.83$ (s, 2H), 7.24 (d, $J=8.4 \mathrm{~Hz}, 2 \mathrm{H}), 7.00(\mathrm{~d}, J=8.4 \mathrm{~Hz}, 2 \mathrm{H})$, 3.94 (q, $J=10.4 \mathrm{~Hz}, 2 \mathrm{H}), 3.72(\mathrm{~s}, 6 \mathrm{H}),{ }^{13} \mathrm{C}$ NMR $(100$ 
$\left.\mathrm{MHz}, \mathrm{CDCl}_{3}\right) \delta: 157.5,142.6,134.7,133.0,131.7,131.6$ $\left(\mathrm{q}, J_{\mathrm{C}-\mathrm{F}}=33.2 \mathrm{~Hz}\right), 129.92 \sim 129.90(\mathrm{~m}), 125.8,123.5(\mathrm{q}$, $\left.J_{\mathrm{C}-\mathrm{F}}=271.2 . \mathrm{Hz}\right), 121.47 \sim 121.4(\mathrm{~m}), 111.5,56.0,30.0$; IR (KBr) v: 3439, 2962, 2837, 1591, 1460, 1377, 1277, 1181, 1130, 1076, 1040, 896, 816, 711, 682, $586 \mathrm{~cm}^{-1}$; HRMS (ESI) calcd for $\mathrm{C}_{33} \mathrm{H}_{21} \mathrm{Br}_{2} \mathrm{~F}_{12} \mathrm{O}_{4}[\mathrm{M}+\mathrm{HCOOH}-$ $\mathrm{H}]^{-}:$868.9600, found 868.9616 .

(R)-6,6'-二溴甲基-3, 3'-二叔丁基-2,2'-二甲氧基-5,5'双 (3,4,5-三氟苯基)联苯 $[(R)-21]$ : 合成步骤与 $(S)-11$ 相 同，产率 78\%. 黄色固体，m.p. $135{ }^{\circ} \mathrm{C}$ (dec.); $[\alpha]_{\mathrm{D}}^{25}$ +19.1 (c 0.215, $\left.\mathrm{CHCl}_{3}\right) ;{ }^{1} \mathrm{H} \mathrm{NMR}\left(400 \mathrm{MHz}, \mathrm{CDCl}_{3}\right) \delta$ : 7.24 (s, 2H), 7.09 (dd, $J=8.0,6.4 \mathrm{~Hz}, 4 \mathrm{H}), 4.26$ (d, $J=$ $10.8 \mathrm{~Hz}, 2 \mathrm{H}), 4.17$ (d, $J=10.4 \mathrm{~Hz}, 2 \mathrm{H}), 3.42$ (s, 6H), 1.42 $(\mathrm{s}, 18 \mathrm{H}) ;{ }^{13} \mathrm{C}$ NMR $\left(100 \mathrm{MHz}, \mathrm{CDCl}_{3}\right) \delta$ : 156.6, 151.0 $\left(\mathrm{ddd}, J_{\mathrm{C}-\mathrm{F}}=249.2,9.9,4.2 \mathrm{~Hz}\right), 144.5,139.4\left(\mathrm{dt}, J_{\mathrm{C}-\mathrm{F}}=\right.$ $250.4,15.2 \mathrm{~Hz}), 136.9 \sim 137.1(\mathrm{~m}), 136.4,133.3,130.7$, 130.0, $113.8\left(\mathrm{dd}, J_{\mathrm{C}-\mathrm{F}}=15.4,5.7 \mathrm{~Hz}\right), 59.8,35.4,30.6$, 30.3; IR (KBr) v: 3443, 2956, 2917, 2869, 1617, 1575, $1527,1466,1434,1419,1380,1361,1265,1236,1207$, 1172, 1047, 979, 906, 858, 765, 707, 631, 582, $557 \mathrm{~cm}^{-1}$; HRMS (ESI) calcd for $\mathrm{C}_{37} \mathrm{H}_{35} \mathrm{Br}_{2} \mathrm{~F}_{6} \mathrm{O}_{4}[\mathrm{M}+\mathrm{HCOOH}-$ $\mathrm{H}^{-}:$: 817.0791, found 817.0808.

$(R)-6,6^{\prime}$-二溴甲基-2,2'-二甲氧基-5,5'-双 $(3,4,5$-三氟 苯基)联苯 $[(R)-22]$ : 合成步骤与 $(S)-11$ 相同, 产率 $96 \%$. 黄色固体, m.p. 78 80 ${ }^{\circ} \mathrm{C} ;{ }^{1} \mathrm{H}$ NMR (400 $\mathrm{MHz} \mathrm{CDCl}_{3}$ ) $\delta: 7.17(\mathrm{~d}, J=8.4 \mathrm{~Hz}, 2 \mathrm{H}), 7.16(\mathrm{dd}, J=8.4,6.8 \mathrm{~Hz}, 4 \mathrm{H})$, $7.04(\mathrm{~d}, J=8.4 \mathrm{~Hz}, 2 \mathrm{H}), 4.08(\mathrm{~s}, 4 \mathrm{H}), 3.78(\mathrm{~s}, 6 \mathrm{H}) ;{ }^{13} \mathrm{C}$ NMR $\left(100 \mathrm{MHz}, \mathrm{CDCl}_{3}\right) \delta: 157.16(\mathrm{~s}), 150.8\left(\mathrm{ddd}, J_{\mathrm{C}-\mathrm{F}}=\right.$ 249.2, 9.9, $4.2 \mathrm{~Hz}), 139.3\left(\mathrm{dt}, J_{\mathrm{C}-\mathrm{F}}=250.2,15.1 \mathrm{~Hz}\right)$, $136.6 \sim 136.4(\mathrm{~m}), 134.5,133.0,131.5,125.7,114.0(\mathrm{dd}$, $\left.J_{\mathrm{C}-\mathrm{F}}=15.4,5.6 \mathrm{~Hz}\right), 111.3,55.9,30.2$.

(S)-4,8-双[3,5-双 (三氟甲基)苯基]-2,10-二叔丁基6,6-二丁基-1,11-二甲氧基-6,7-二氢-5H-二苯并 $[c, e]$ 氮杂 草-6-溴化铵 $[(S)-13]$ : 室温下, 向光学纯的催化剂中间 体 $(S)$-11 (355.9 mg, $0.38 \mathrm{mmol}$ )的乙腈 $(4.0 \mathrm{~mL}$ )溶液中加 入正二丁胺 $(57.6 \mu \mathrm{L}, 0.342 \mathrm{mmol})$ 和碳酸钾 $(655 \mathrm{mg}, 3.8$ $\mathrm{mmol}$ ), 在 $85{ }^{\circ} \mathrm{C}$ 反应过夜. 反应结束后, 将反应体系冷 却至室温, 加二氯甲烷稀释, 用硅藻土过滤. 滤液浓缩 后所得的粗产物经硅胶柱层析(甲醇/二氯甲烷，1:80 1：20)分离纯化, 得 $245.4 \mathrm{mg}$ 手性季铵盐目标物(S)-13, 收率 66\%. 黄色固体, m.p. 229 231 ${ }^{\circ} \mathrm{C}$; $[\alpha]_{\mathrm{D}}^{25}-21.4(c$ $\left.0.41, \mathrm{CHCl}_{3}\right) ;{ }^{1} \mathrm{H} \mathrm{NMR}\left(400 \mathrm{MHz}\right.$, acetone- $\left.d_{6}\right) \delta: 8.48(\mathrm{br}$ $\mathrm{s}, 4 \mathrm{H}), 8.12(\mathrm{~s}, 2 \mathrm{H}), 7.51(\mathrm{~s}, 2 \mathrm{H}), 4.61(\mathrm{~d}, J=14.0 \mathrm{~Hz}, 2 \mathrm{H})$, $4.42(\mathrm{~d}, J=14.0 \mathrm{~Hz}, 2 \mathrm{H}), 3.36(\mathrm{~s}, 6 \mathrm{H}), 3.28 \sim 3.22(\mathrm{~m}$,
2H), $2.82(\mathrm{td}, J=13.2,4.0 \mathrm{~Hz}, 2 \mathrm{H}), 1.50(\mathrm{~s}, 18 \mathrm{H}), 1.30 \sim$ $1.34(\mathrm{~m}, 2 \mathrm{H}), 1.08 \sim 1.17(\mathrm{~m}, 2 \mathrm{H}), 0.84 \sim 0.89(\mathrm{~m}, 2 \mathrm{H})$, $0.61(\mathrm{t}, J=7.2 \mathrm{~Hz}, 6 \mathrm{H}), 0.57 \sim 0.51(\mathrm{~m}, 2 \mathrm{H}) ;{ }^{13} \mathrm{C}$ NMR $\left(100 \mathrm{MHz}\right.$, acetone- $\left.d_{6}\right) \delta: 160.0,146.1,143.9,136.4$, $132.2,131.2,125.5,124.41\left(\mathrm{q}, J_{\mathrm{C}-\mathrm{F}}=270.9 \mathrm{~Hz}\right), 122.6 \sim$ 122.3 (m), 61.8, 59.2, 58.2, 36.1, 30.6, 24.9, 19.7, 13.9; IR (KBr) v: 3401, 3193, 2962, 2879, 1617, 1470, 1386, 1361, 1329, 1277, 1233, 1185, 1143, 1111, 1063, 906, 848, 717, $682,614 \mathrm{~cm}^{-1}$; HRMS (ESI) calcd for $\mathrm{C}_{48} \mathrm{H}_{54} \mathrm{~F}_{12} \mathrm{NO}_{2}[\mathrm{M}-$ $\mathrm{Br}]^{+}$: 904.3957; found 904.3939.

(S)-4,8-双[3,5-双 (三氟甲基)苯基]-6,6-二丁基-1,11-二 甲氧基 - 6,7 -二氢 $-5 H$-二苯并 $[c, e]$ 氮杂草 -6- 溴化铵 [(S)-14]: 合成步骤与催化剂 $(S)-13$ 相同, 产率 $82 \%$. 黄 色固体, m.p. $227 \sim 229{ }^{\circ} \mathrm{C}$; $[\alpha]_{\mathrm{D}}^{25}-21.8\left(c 0.675, \mathrm{CHCl}_{3}\right)$; ${ }^{1} \mathrm{H}$ NMR (400 MHz, $\left.\mathrm{CDCl}_{3}\right) \delta: 7.95$ (s, 6H), 7.48 (d, $J=$ $8.8 \mathrm{~Hz}, 2 \mathrm{H}), 7.32$ (d, $J=8.8 \mathrm{~Hz}, 2 \mathrm{H}), 4.54$ (d, $J=14.0 \mathrm{~Hz}$, 2H), $3.94(\mathrm{~s}, 6 \mathrm{H}), 3.90(\mathrm{~d}, J=14.0 \mathrm{~Hz}, 2 \mathrm{H}), 3.14(\mathrm{t}, J=$ $12.8 \mathrm{~Hz}, 2 \mathrm{H}), 2.68(\mathrm{td}, J=12.8,4.0 \mathrm{~Hz}, 2 \mathrm{H}), 1.04 \sim 1.02$ $(\mathrm{m}, 4 \mathrm{H}), 0.82 \sim 0.76(\mathrm{~m}, 2 \mathrm{H}), 0.58(\mathrm{t}, J=7.2 \mathrm{~Hz}, 6 \mathrm{H})$, $0.22 \sim 0.16(\mathrm{~m}, 2 \mathrm{H}) ;{ }^{13} \mathrm{C}$ NMR $\left(100 \mathrm{MHz}, \mathrm{CDCl}_{3}\right) \delta$ : $157.4,141.4,133.0,132.4,130.0 \sim 129.8(\mathrm{~m}), 126.6$, $124.8,122.7\left(\mathrm{q}, J_{\mathrm{C}-\mathrm{F}}=271.4 \mathrm{~Hz}\right), 121.5 \sim 121.4(\mathrm{~m})$, 113.8, 57.3, 57.2, 56.1, 24.2, 18.9, 13.1; IR (KBr) v: 3407, 2959, 2879, 2840, 1614, 1591, 1498, 1463, 1380, 1277, $1175,1137,1105,1089,1047,951,903,816,717,682 \mathrm{~cm}$

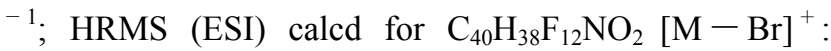
792.2716, found 792.2708.

(R)-2,10-二叔丁基-6,6-二丁基-1,11-二甲氧基-4,8双( $3,4,5$-三氟苯基)-6,7-二氢- $5 H$-二苯并 $[c, e]$ 氮杂草- 6 -溴 化铵 $[(R)-23]$ : 合成步骤与催化剂 $(S)-13$ 相同, 产率 $80 \%$. 黄色固体， m.p. $232 \sim 234{ }^{\circ} \mathrm{C} ;[\alpha]_{\mathrm{D}}^{25}+25.9$ (c 0.21 , $\mathrm{CHCl}_{3}$ ); ${ }^{1} \mathrm{H}$ NMR (400 MHz, acetone- $\left.d_{6}\right) \delta: 7.66$ (br s, 4H), 7.46 (s, 2H), 4.81 (d, $J=14.0 \mathrm{~Hz}, 2 \mathrm{H}), 4.04$ (d, $J=$ $14.0 \mathrm{~Hz}, 2 \mathrm{H}), 3.27(\mathrm{~s}, 6 \mathrm{H}), 3.23 \sim 3.17(\mathrm{~m}, 2 \mathrm{H}), 2.91 \sim$ $2.84(\mathrm{~m}, 2 \mathrm{H}), 1.47(\mathrm{~s}, 18 \mathrm{H}), 1.30 \sim 1.33(\mathrm{~m}, 2 \mathrm{H}), 1.20 \sim$ $1.10(\mathrm{~m}, 2 \mathrm{H}), 0.95 \sim 1.04(\mathrm{~m}, 2 \mathrm{H}), 0.71(\mathrm{t}, J=7.2 \mathrm{~Hz}, 6 \mathrm{H})$, $0.42 \sim 0.53(\mathrm{~m}, 2 \mathrm{H}) ;{ }^{13} \mathrm{C}$ NMR $\left(100 \mathrm{MHz}\right.$, acetone- $\left.d_{6}\right) \delta$ : $159.4,153.3 \sim 152.3(\mathrm{~m}), 150.6 \sim 150.0(\mathrm{~m}), 145.9,140.1$ $\left(\mathrm{dt}, \quad J_{\mathrm{C}-\mathrm{F}}=248.6,15.2 \mathrm{~Hz}\right), 137.5 \sim 137.3(\mathrm{~m}), 136.5$, $132.0,130.5,125.3,117.0 \sim 115.1(\mathrm{~m}), 61.4,58.4,57.9$, 35.9, 30.4, 25.1, 19.9, 13.6; IR (KBr) v: 3417, 2962, 2873, $1614,1585,1527,1473,1428,1406,1271,1249$, 1229, 1079, 1044, 973, 871, 855, 771, 720, 634, 589, $531 \mathrm{~cm}^{-1}$; HRMS (ESI) calcd for $\mathrm{C}_{44} \mathrm{H}_{52} \mathrm{~F}_{6} \mathrm{NO}_{2}[\mathrm{M}-\mathrm{Br}]^{+}:$740.3907, 
found 740.3897 .

$(R)-6,6$-二丁基-1,11-二甲氧基-4,8-双(3,4,5-三氟苯 基)-6,7-二氢-5H-二苯并 $[c, e]$ 氮杂草-6-溴化铵 $[(R)-24]^{[35]}$ : 合成步骤与催化剂 $(S)-13$ 相同, 黄色固体手性季铵盐, 产率 81\%. m.p. 230 232 ${ }^{\circ} \mathrm{C}$; ${ }^{1} \mathrm{H}$ NMR $(400 \mathrm{MHz}$, $\left.\mathrm{CDCl}_{3}\right) \delta: 7.41(\mathrm{~d}, J=8.8 \mathrm{~Hz}, 2 \mathrm{H}), 7.24(\mathrm{~d}, J=8.8 \mathrm{~Hz}$, 2H), 7.17 (br s, 4H), 4.74 (d, $J=14.0 \mathrm{~Hz}, 2 \mathrm{H}), 3.89$ (s, $6 \mathrm{H}), 3.64(\mathrm{~d}, J=14.0 \mathrm{~Hz}, 2 \mathrm{H}), 3.18$ (t, $J=12.8 \mathrm{~Hz}, 2 \mathrm{H})$, $2.72 \sim 2.67(\mathrm{~m}, 2 \mathrm{H}), 1.10 \sim 1.09(\mathrm{~m}, 4 \mathrm{H}), 0.99 \sim 0.95(\mathrm{~m}$, $2 \mathrm{H}), 0.72(\mathrm{t}, J=6.8 \mathrm{~Hz}, 6 \mathrm{H}), 0.29 \sim 0.26(\mathrm{~m}, 2 \mathrm{H}) ;{ }^{13} \mathrm{C}$ NMR (100 MHz, $\left.\mathrm{CDCl}_{3}\right) \delta: 156.9,152.2 \sim 152.0(\mathrm{~m})$, $149.8 \sim 149.5(\mathrm{~m}), 139.3\left(\mathrm{dt}, J_{\mathrm{C}-\mathrm{F}}=252.3,15.1 \mathrm{~Hz}\right)$, $135.0 \sim 134.8(\mathrm{~m}), 132.5,132.0,126.4,125.1,115.0 \sim$ $113.9(\mathrm{~m}), 113.3,57.2,56.9,55.9,24.3,19.1,13.0$.

(S)-4,8-双 [3,5-双(三氟甲基)苯基]-2,10-二叔丁基6,6 -二丁基-1,11-二羟基-6,7-二氢- $5 H$-二苯并 $[c, e]$ 氮杂草 -6-溴化铵 $[(S)-15]$ : 参照文献 $[64]$ 方法. 在 $-78{ }^{\circ} \mathrm{C}$ 及氩 气保护的条件下, 向催化剂 $(S)-\mathbf{1 3}(118.2 \mathrm{mg}, 0.12 \mathrm{mmol})$ 的无水二氯甲烷 $(1.0 \mathrm{~mL})$ 溶液中逐滴滴加 $1 \mathrm{~mol} / \mathrm{L}$ 三溴 化嗍(0.84 mL, $0.84 \mathrm{mmol})$, 反应体系升至室温后, 在室 温下反应 $4 \mathrm{~h}$. 将反应体系倒入冰水中, 用二氯甲烷萃 取三次, 合并后的有机相, 用无水硫酸钠干燥, 过滤, 减压浓缩. 得到的粗产品经硅胶柱层析(甲醇/二氯甲烷, $V: V=1: 40 \sim 1: 5)$ 分离纯化得 $97.5 \mathrm{mg}(S)-15$, 收率 $81 \%$. 白色固体, m.p. $150 \sim 152{ }^{\circ} \mathrm{C}$; $[\alpha]_{\mathrm{D}}^{25}+6$ (c 0.50 , $\mathrm{CHCl}_{3}$ ); ${ }^{1} \mathrm{H}$ NMR (400 MHz, acetone- $\left.d_{6}\right) \delta: 8.35$ (s, 2H), $8.22(\mathrm{~s}, 2 \mathrm{H}), 8.07(\mathrm{~s}, 2 \mathrm{H}), 7.36(\mathrm{~s}, 2 \mathrm{H}), 4.54$ (d, $J=13.6$ $\mathrm{Hz}, 2 \mathrm{H}), 3.67$ (d, $J=13.2 \mathrm{~Hz}, 2 \mathrm{H}), 3.05 \sim 2.98(\mathrm{~m}, 2 \mathrm{H})$, $2.82(\mathrm{td}, J=13.2,4.4 \mathrm{~Hz}, 2 \mathrm{H}), 1.53(\mathrm{~s}, 18 \mathrm{H}), 1.25 \sim 1.19$ $(\mathrm{m}, 2 \mathrm{H}), 1.11 \sim 1.02(\mathrm{~m}, 2 \mathrm{H}), 0.93 \sim 0.86(\mathrm{~m}, 2 \mathrm{H}), 0.61(\mathrm{t}$, $J=7.2 \mathrm{~Hz}, 6 \mathrm{H}), 0.47 \sim 0.35(\mathrm{~m}, 2 \mathrm{H}) ;{ }^{13} \mathrm{C} \mathrm{NMR}(100 \mathrm{MHz}$, acetone- $\left.d_{6}\right) \quad \delta: 159.8,145.3,142.8,131.9 \sim 131.7(\mathrm{~m})$, $131.5,131.3 \sim 131.1(\mathrm{~m}), 130.3,129.7,124.43,124.40$ (q, $\left.J_{\mathrm{C}-\mathrm{F}}=270.6 \mathrm{~Hz}\right), 121.5 \sim 121.3(\mathrm{~m}), 58.6,57.4,35.9,30.0$, 24.96, 19.7, 13.7; IR (KBr) v: 3436, 2959, 2869, 1614, 1575, 1473, 1434, 1377, 1361, 1281, 1181, 1130, 1105, 893, 848, 714, 678, 627, $592 \mathrm{~cm}^{-1}$; HRMS (ESI) calcd for $\mathrm{C}_{46} \mathrm{H}_{50} \mathrm{~F}_{12} \mathrm{NO}_{2}[\mathrm{M}-\mathrm{Br}]^{+}:$876.3655, found 876.3644.

(S)-4,8-双[3,5-双(三氟甲基)苯基]-6,6-二丁基-1,11二羟基 $-6,7-$ 二氢 $-5 H$-二苯并 $[c, e]$ 氮杂草 -6 - 溴化铵 $[(S)-16]$ : 合成步骤与催化剂 $(S)-15$ 相同, 产率 85\%. 黄 色固体, m.p. $265{ }^{\circ} \mathrm{C}$ (dec.); $[\alpha]_{\mathrm{D}}^{25}-55$ (c 0.20, DMF); ${ }^{1} \mathrm{H}$ NMR (400 MHz, MeOD- $\left.d_{4}\right) \delta: 8.09$ (s, 6H), 7.44 (d, $J=$ $8.4 \mathrm{~Hz}, 2 \mathrm{H}), 7.29$ (d, $J=8.8 \mathrm{~Hz}, 2 \mathrm{H}), 4.42$ (d, $J=14.0 \mathrm{~Hz}$,
2H), $3.73(\mathrm{~d}, J=13.6 \mathrm{~Hz}, 2 \mathrm{H}), 2.92 \sim 2.86(\mathrm{~m}, 2 \mathrm{H}), 2.62$ $(\mathrm{td}, J=12.8,4.4 \mathrm{~Hz}, 2 \mathrm{H}), 1.11 \sim 0.92(\mathrm{~m}, 4 \mathrm{H}), 0.85 \sim 0.72$ (m, 2H), $0.60(\mathrm{t}, J=7.2 \mathrm{~Hz}, 6 \mathrm{H}), 0.24 \sim 0.11(\mathrm{~m}, 2 \mathrm{H}) ;{ }^{13} \mathrm{C}$ NMR (100 MHz, MeOD- $\left.d_{4}\right) \delta: 157.0,143.8,133.9,133.4$, 131.7, 126.7, 126.5, 124.6 (q, $\left.J_{\mathrm{C}-\mathrm{F}}=270.6 \mathrm{~Hz}\right), 122.7 \sim$ 122.5 (m), 119.8, 58.2, 58.0, 25.4, 20.1, 13.8; IR (KBr) v: 3423, 2969, 2917, 2882, 2847, 1591, 1460, 1377, 1281, 1249, 1178, 1143, 1101, 1047, 900, 835, 714, $682 \mathrm{~cm}^{-1}$; HRMS (ESI) calcd for $\mathrm{C}_{38} \mathrm{H}_{34} \mathrm{~F}_{12} \mathrm{NO}_{2}[\mathrm{M}-\mathrm{Br}]^{+}$: 764.2392, found 764.2390 .

(R)-2,10-二叔丁基-6,6-二丁基-1,11-二羟基-4,8-双 (3,4,5-三氟苯基)- 6,7 -二氢- $5 H$-二苯并 $[c, e]$ 氮杂草-6-溴化 铵 $[(R)-25]$ : 合成步骤与催化剂 $(S)-15$ 相同, 产率 $57 \%$. 黄色固体，m.p. $175 \sim 177{ }^{\circ} \mathrm{C} ;[\alpha]_{\mathrm{D}}^{25}+6.5$ (c 0.195 , $\mathrm{CHCl}_{3}$ ); ${ }^{1} \mathrm{H}$ NMR (400 MHz, acetone- $\left.d_{6}\right) \delta: 7.53$ ( br s, 2H), 7.32 (br s, 2H), 7.25 (s, 2H), 4.63 (d, J=13.2 Hz, 2H), 3.42 (d, $J=13.2 \mathrm{~Hz}, 2 \mathrm{H}), 3.02$ (t, $J=12.4 \mathrm{~Hz}, 2 \mathrm{H})$, $2.87(\mathrm{td}, J=12.8,4.4 \mathrm{~Hz}, 2 \mathrm{H}), 1.50(\mathrm{~s}, 18 \mathrm{H}), 1.22 \sim 1.06$ $(\mathrm{m}, 6 \mathrm{H}), 0.73(\mathrm{t}, J=7.2 \mathrm{~Hz}, 6 \mathrm{H}), 0.45 \sim 0.37(\mathrm{~m}, 2 \mathrm{H}) ;{ }^{13} \mathrm{C}$ NMR $\left(100 \mathrm{MHz}\right.$, acetone- $\left.d_{6}\right) \delta: 159.9,152.5 \sim 151.5(\mathrm{~m})$, $150.0 \sim 149.0(\mathrm{~m}), 141.3,138.8 \sim 138.6(\mathrm{~m}), 138.6(\mathrm{dt}$, $\left.J_{\mathrm{C}^{-\mathrm{F}}}=247.6,15.1 \mathrm{~Hz}\right), 130.5,128.7,128.0,123.3,115.2$ 14.3 (m), 35.0, 29.1, 24.3, 19.1, 12.7; IR (KBr) v: 3417, 2959, 2872, 1614, 1575, 1530, 1466, 1434, 1383, 1364, 1306, 1245, 1197, 1044, 864, 852, 765, 707, 688, 653, 608, $554 \mathrm{~cm}^{-1}$; HRMS (ESI) calcd for $\mathrm{C}_{42} \mathrm{H}_{48} \mathrm{~F}_{6} \mathrm{NO}_{2}[\mathrm{M}-\mathrm{Br}]^{+}$: 712.3594 , found 712.3588 .

(R)-6,6-二丁基-1,11-二羟基-4,8-双 $(3,4,5$-三氟苯 基)-6,7-二氢-5H-二苯并 $[c, e]$ 氮杂草-6-溴化铵 $[(R)-26]$ : 合成步骤与催化剂 $(S)-15$ 相同, 产率 $50 \%$. 黄色固体, m.p. $250{ }^{\circ} \mathrm{C}$ (dec.); $[\alpha]_{\mathrm{D}}^{25}+24.7$ (c $\left.0.39, \mathrm{CH}_{3} \mathrm{OH}\right) ;{ }^{1} \mathrm{H}$ NMR (400 MHz, MeOD-d $d_{4} \delta: 7.38$ (d, $\left.J=8.8 \mathrm{~Hz}, 4 \mathrm{H}\right)$, $7.22(\mathrm{~d}, J=8.8 \mathrm{~Hz}, 4 \mathrm{H}), 4.56$ (d, $J=13.6 \mathrm{~Hz}, 2 \mathrm{H}), 3.54$ (d, $J=13.6 \mathrm{~Hz}, 2 \mathrm{H}), 2.92$ (t, $J=12.8 \mathrm{~Hz}, 2 \mathrm{H}), 2.67$ (td, $J=12.8,4.4 \mathrm{~Hz}, 2 \mathrm{H}), 1.15 \sim 1.03(\mathrm{~m}, 4 \mathrm{H}), 1.01 \sim 0.90(\mathrm{~m}$, $2 \mathrm{H}), 0.75(\mathrm{t}, J=7.2 \mathrm{~Hz}, 6 \mathrm{H}), 0.25 \sim 0.19(\mathrm{~m}, 2 \mathrm{H}) ;{ }^{13} \mathrm{C}$ NMR $\left(100 \mathrm{MHz}, \mathrm{MeOD}-d_{4}\right) \delta: 156.6,153.9 \sim 153.4(\mathrm{~m})$, $151.4 \sim 150.9(\mathrm{~m}), 140.6\left(\mathrm{dt}, J_{\mathrm{C}-\mathrm{F}}=250.0,15.0 \mathrm{~Hz}\right)$, $137.8 \sim 137.6(\mathrm{~m}), 133.6,133.2,126.8,126.4,119.5$, $116.1 \sim 115.7(\mathrm{~m}), 57.95,57.9,25.5,20.3,13.6$; IR $(\mathrm{KBr})$ v: 3340, 3167, 2969, 2940, 2876, 2568, 2376, 1902, 1617, 1598, 1527, 1492, 1466, 1441, 1418, 1364, 1306, 1290, 1239, 1210, 1181, 1133, 1050, 1021, 1005, 874, 855, 845, $810,752,730,711,679,656,631,563,534 \mathrm{~cm}^{-1}$; HRMS 
(ESI) calcd for $\mathrm{C}_{34} \mathrm{H}_{32} \mathrm{~F}_{6} \mathrm{NO}_{2}[\mathrm{M}-\mathrm{Br}]^{+}: 600.2331$, found 600.2340 .

\section{4 相转移催化剂催化的不对称烷基化反应}

在 $0{ }^{\circ} \mathrm{C}$ 及氩气保护条件下, 向 $N$-二苯基甲亚基甘 氨酸叔丁酯 29 (90.4 mg, $0.3 \mathrm{mmol}$ )和手性相转移催化剂 $(0.003 \mathrm{mmol})$ 的甲苯 $(1.5 \mathrm{~mL})$ 混合液中, 逐滴滴加茮溴 $(109 \mu \mathrm{L}, 0.9 \mathrm{mmol})$ 和 $50 \%$ 的 $\mathrm{KOH}$ 水溶液 $(0.5 \mathrm{~mL})$, 并在 剧烈摚拌下进行催化反应. 反应完成后, 将反应混合物 倒入水中, 用二氯甲烷萃取, 有机相用无水硫酸钠干燥, 过滤, 减压浓缩. 得到的粗产品经硅胶柱层析(正己烷/ 二氯甲烷/乙酸乙酯, 体积比 $100: 1: 1 \sim 70: 1: 1)$ 分 离纯化后得到目标化合物 $N$-二苯基甲亚基苯丙氨酸叔 丁酯 $(30){ }^{[35]}$. ${ }^{1} \mathrm{H}$ NMR (400 $\left.\mathrm{MHz}, \mathrm{CDCl}_{3}\right) \delta: 7.58 \sim 7.56$ $(\mathrm{m}, 2 \mathrm{H}), 7.38 \sim 7.25(\mathrm{~m}, 6 \mathrm{H}), 7.21 \sim 7.13(\mathrm{~m}, 3 \mathrm{H}), 7.06 \sim$ $7.04(\mathrm{~m}, 2 \mathrm{H}), 6.60(\mathrm{~d}, J=6.8 \mathrm{~Hz}, 2 \mathrm{H}), 4.11$ (dd, $J=9.2$, $4.4 \mathrm{~Hz}, 1 \mathrm{H}), 3.23$ (dd, $J=13.6,4.4 \mathrm{~Hz}, 1 \mathrm{H}), 3.16$ (dd, $J=$ 13.2, $9.2 \mathrm{~Hz}, 1 \mathrm{H}), 1.44(\mathrm{~s}, 9 \mathrm{H}) ;{ }^{13} \mathrm{C}$ NMR $(100 \mathrm{MHz}$, $\left.\mathrm{CDCl}_{3}\right) \delta: 171.0,170.4,139.7,138.5,136.5,130.2,130.0$, $128.9,128.3,128.2,128.2,128.1,127.8,126.3,81.3,68.1$, 39.7, 28.2; HPLC: Diacel Chiralpak OD-H (5 $\mu \mathrm{m}, 4.6$ $\mathrm{mm} \times 250 \mathrm{~mm}), V($ hexane $) / V(i-\mathrm{PrOH})=100 / 1$, flow rate $=$ $0.3 \mathrm{~mL} / \mathrm{min}, \lambda=254 \mathrm{~nm}$, retention time: $21.7 \mathrm{~min}$ [(R)-enantiomer], $31.0 \mathrm{~min}[(S)$-enantiomer].

辅助材料(Supporting Information) 催化剂及催化剂 中间体的 ${ }^{1} \mathrm{H}$ NMR, ${ }^{13} \mathrm{C}$ NMR, HRMS 和 HPLC 的谱图. 这些材料可以免费从本刊网站(http://sioc-journal.cn/)上 下载.

\section{References}

[1] Odonnell, M. J.; Bennett, W. D.; Wu, S. D. J. Am. Chem. Soc. 1989, $111,2353$.

[2] Corey, E. J.; Xu, F.; Noe, M. C. J. Am. Chem. Soc. 1997, 119, 12414.

[3] Lygo, B.; Wainwright, P. G. Tetrahedron Lett. 1997, 38, 8595.

[4] Jew, S. S.; Jeong, B. S.; Yoo, M. S.; Huh, H.; Park, H. G. Chem. Commun. 2001, 1244.

[5] Nakoji, M.; Kanayama, T.; Okino, T.; Takemoto, Y. Org. Lett. 2001, 3,3329 .

[6] Kita, T.; Georgieva, A.; Hashimoto, Y.; Nakata, T.; Nagasawa, K. Angew. Chem., Int. Ed. 2002, 41, 2832.

[7] Liu, X.; Dong, S.; Lin, L.; Feng, X. Chin. J. Chem. 2018, 36, 791.

[8] Wang, P.-S.; Chen, D.-F.; Gong, L.-Z. Top. Curr. Chem. 2019, 378 , 9.

[9] Tang, Q.; Fu, K.; Ruan, P.; Dong, S.; Su, Z.; Liu, X.; Feng, X. Angew. Chem., Int. Ed. 2019, 58, 11846.

[10] You, S.; Cheng, Y.-Z.; Zhao, Q.-R.; Zhang, X.; You, S.-L. Angew. Chem., Int. Ed. 2019, 58, 18069.

[11] Li, S.; Xiang, S.-H.; Tan, B. Chin. J. Chem. 2020, 38, 213.

[12] Yang, G.; Zhang, W. Chem. Soc. Rev. 2018, 47, 1783.

[13] Wang, H. Catalysts 2019, 9, 244.
[14] Guo, W.; Liu, X.; Liu, Y.; Li, C. ACS Catal. 2018, 8, 328.

[15] Qian, D.; Sun, J. Chem.-Eur. J. 2019, 25, 3740.

[16] Jiang, F.; Chen, K.-W.; Wu, P.; Zhang, Y.-C.; Shi, F.; Jiao, Y.; Jiang, F.; Chen, K.-W.; Wu, P.; Zhang, Y.-C.; Jiao, Y.; Shi, F. Angew. Chem., Int. Ed. 2019, 58, 15104

[17] Park, H. G.; Jeong, B. S.; Yoo, M. S.; Lee, J. H.; Park, M. K.; Lee, Y. J.; Kim, M. J.; Jew, S. S. Angew. Chem., Int. Ed. 2002, 41, 3036.

[18] Belokon, Y. N.; Bespalova, N. B.; Churkina, T. D.; Cisarova, I.; Ezernitskaya, M. G.; Harutyunyan, S. R.; Hrdina, R.; Kagan, H. B.; Kocovsky, P.; Kochetkov, K. A.; Larionov, O. V.; Lyssenko, K. A.; North, M.; Polasek, M.; Peregudov, A. S.; Prisyazhnyuk, V. V.; Vyskocil, S. J. Am. Chem. Soc. 2003, 125, 12860.

[19] Lygo, B.; Andrews, B. I. Acc. Chem. Res. 2004, 37, 518.

[20] O'Donnell, M. J. Acc. Chem. Res. 2004, 37, 506.

[21] Kitamra, M.; Shirakawa, S.; Maruoka, K. Angew. Chem., Int. Ed. 2005, 44, 1549.

[22] Ooi, T.; Kameda, M.; Maruoka, K. J. Am. Chem. Soc. 1999, 121, 6519.

[23] Ooi, T.; Takeuchi, M.; Kameda, M.; Maruoka, K. J. Am. Chem. Soc. 2000, 122, 5228.

[24] Ooi, T.; Takahashi, M.; Doda, K.; Maruoka, K. J. Am. Chem. Soc. 2002, 124,7640 .

[25] Ooi, T.; Kameda, M.; Maruoka, K. J. Am. Chem. Soc. 2003, 125, 5139.

[26] Ooi, T.; Takeuchi, M.; Kato, D.; Uematsu, Y.; Tayama, E.; Sakai, D.; Maruoka, K. J. Am. Chem. Soc. 2005, 127, 5073.

[27] Shirakawa, S.; Yamamoto, K.; Kitamura, M.; Ooi, T.; Maruoka, K. Angew. Chem., Int. Ed. 2005, 44, 625.

[28] Freedman, H. H. Pure Appl. Chem. 1986, 58, 857.

[29] Hashimoto, T.; Maruoka, K. Chem. Rev. 2007, 107, 5656.

[30] Shirakawa, S.; Maruoka, K. Angew. Chem., Int. Ed. 2013, 52, 4312.

[31] Hashimoto, T.; Sakata, K.; Tamakuni, F.; Dutton, M. J.; Maruoka, K. Nat. Chem. 2013, 5, 240.

[32] Inukai, T.; Kano, T.; Maruoka, K. Angew. Chem., Int. Ed. 2020, 59, 2211.

[33] Wang, Y.; Ye, J.; Liang, X. Adv. Synth. Catal. 2007, 349, 1033.

[34] Wang, Y.-G.; Maruoka, K. Org. Process Res. Dev. 2007, 11, 628.

[35] Wang, Y.-G.; Ueda, M.; Wang, X.; Han, Z.; Maruoka, K. Tetrahedron 2007, 63, 6042 .

[36] Ooi, T.; Uematsu, Y.; Kameda, M.; Maruoka, K. Angew. Chem., Int. Ed. 2002, 41, 1551.

[37] He, R.; Wang, X.; Hashimoto, T.; Maruoka, K. Angew. Chem., Int. Ed. 2008, 47, 9466 .

[38] He, R.; Ding, C.; Maruoka, K. Angew. Chem., Int. Ed. 2009, 48, 4559.

[39] He, R.; Shirakawa, S.; Maruoka, K. J. Am. Chem. Soc. 2009, 131, 16620 .

[40] Liu, Y.; Usui, A.; Shirakawa, S.; Maruoka, K. Asian J. Org. Chem. 2012, 1,180

[41] Shirakawa, S.; Liu, Y.; Usui, A.; Maruoka, K. ChemCatChem 2012, 4,980 .

[42] Ooi, T.; Fujioka, S.; Maruoka, K. J. Am. Chem. Soc. 2004, 126, 11790

[43] Kano, T.; Yamaguchi, Y.; Maruoka, K. Angew. Chem., Int. Ed. 2009, 48, 1838.

[44] Kano, T.; Sakamoto, R.; Yamaguchi, Y.; Itoh, K.; Maruoka, K. Chem. Commun. 2013, 49, 1118

[45] Kano, T.; Maruyama, H.; Akiyama, K.; Maruoka, K. Tetrahedron Lett. 2014, 55, 4227.

[46] Kano, T.; Kobayashi, R.; Maruoka, K. Angew. Chem., Int. Ed. 2015, $54,8471$.

[47] Liu, Y.; Arumugam, N.; Almansour, A. I.; Kumar, R. S.; Maruoka, K. Chem. Rec. 2017, 17, 1059.

[48] Ooi, T.; Taniguchi, M.; Kameda, M.; Maruoka, K. Angew. Chem., Int. Ed. 2002, 41, 4542 .

[49] Ooi, T.; Kameda, M.; Taniguchi, M.; Maruoka, K. J. Am. Chem. Soc. 2004, 126, 9685.

[50] Ooi, T.; Maruoka, K. Acc. Chem. Res. 2004, 37, 526. 
[51] Kano, T.; Yamamoto, A.; Song, S.; Maruoka, K. Bull. Chem. Soc. Jpn. 2011, 84, 1057.

[52] Kano, T.; Yamamoto, A.; Song, S.; Maruoka, K. Chem. Commun. 2011, 47, 4358.

[53] Ooi, T.; Uematsu, Y.; Maruoka, K. J. Am. Chem. Soc. 2006, 128, 2548.

[54] Ooi, T.; Uematsu, Y.; Fujimoto, J.; Fukumoto, K.; Maruoka, K. Tetrahedron Lett. 2007, 48, 1337.

[55] Wang, Y.-G.; Kumano, T.; Kano, T.; Maruoka, K. Org. Lett. 2009, $11,2027$.

[56] Wang, Y.-G.; Mii, H.; Kano, T.; Maruoka, K. Bioorg. Med. Chem. Lett. 2009, 19, 3795.

[57] Kubota, Y.; Shirakawa, S.; Inoue, T.; Maruoka, K. Tetrahedron Lett. 2012, 53, 3739 .

[58] Shirakawa, S.; Yamamoto, K.; Maruoka, K. Angew. Chem., Int. Ed.
2015, 54, 838

[59] Kubota, Y.;Shirakawa, S.; Inoue, T.; Maruoka, K. Tetrahedron Lett. 2012, 53, 3739.

[60] Kozel, V.; Daniliuc, C. D.; Kirsch, P.; Haufe, G. Angew. Chem., Int. Ed. 2017, 56, 15456 .

[61] Costantino, A. R.; Ocampo, R. A.; Montiel Schneider, M. G.; Fernandez, G.; Koll, L. C.; Mandolesi, S. D. Synth. Commun. 2013, 43, 3192.

[62] Hattori, T.; Shimazumi, Y.; Goto, H.; Yamabe, O.; Morohashi, N.; Kawai, W.; Miyano, S. J. Org. Chem. 2003, 68, 2099.

[63] Edsall, R. J.; Harris, H. A.; Manas, E. S.; Mewshaw, R. E. Bioorg Med. Chem. 2003, 11, 3457.

Bai, S.; Li, S.; Xu, J.; Peng, X.; Sai, K.; Chu, W.; Tu, Z.; Zeng, C.; Mach, R. H. J. Med. Chem. 2014, 57, 4239.

(Li, L.) 